\title{
DAAD Studien
}

\section{Studienvorbereitung und -einstieg internationaler}

Studierender in Deutschland MASSNAHMEN, STRUKTUREN UND PRAXISBEISPIELE 


\section{Herausgeber DAAD}

DAAD

Deutscher Akademischer Austauschdienst

German Academic Exchange Service

Kennedyallee 50, D-53175 Bonn

Bereich S1 Strategie und Steuerung

\section{Autoren}

Dr. Jesús Pineda

Maike Rech

Gestaltung und Satz DITHO Design GmbH, Köln

Auflage Juni 2020

DOI http://doi.org/10.46685/DAADStudien.2020.01

Ein herzliches Dankeschön an Benedikt Tränkle für seine Unterstützung bei der Durchführung der Untersuchung sowie dem Verfassen der Publikation. Zur Erstellung der Textabschnitte zum Hochschulzugang und -zulassung internationaler Studierender in Deutschland wurden die Autoren von Ursula Wittersheim und Katharina Maschke (Referat S11-Strategieentwicklung und Hochschulpolitik) inhaltlich unterstützt. Für die freundliche Unterstützung sei beiden Kolleginnen hiermit herzlich gedankt.

(c) DAAD

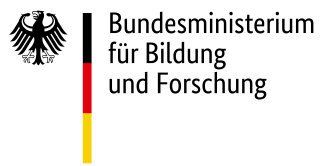

Diese Publikation wird aus Zuwendungen des Bundesministeriums für Bildung und Forschung (BMBF) an den DAAD finanziert. Die Verantwortung für den Inhalt dieser Veröffentlichung liegt bei den Autoren. 
Studienvorbereitung und -einstieg internationaler Studierender in Deutschland MASSNAHMEN, STRUKTUREN UND PRAXISBEISPIELE Jesús Pineda und Maike Rech 



\section{EXECUTIVE SUMMARY}

Internationale Studierende sind eine wachsende Gruppe an deutschen Hochschulen, welche im Vergleich zu deutschen Studierenden mit besonderen Herausforderungen konfrontiert wird. Schätzungen des Deutschen Zentrums für Hochschul- und Wissenschaftsforschung (DZHW) zufolge brechen $45 \%$ der internationalen Studierenden im Bachelor und $29 \%$ im Master ihr Studium ab. Mehrere Analysen haben gezeigt, dass diese Studierendengruppe neben Sprachbarrieren und finanziellen Herausforderungen aufgrund ihres Bildungshintergrunds möglicherweise nicht über die notwendige studienbezogene Vorbereitung für ein Hochschulstudium in Deutschland verfügt. Im Allgemeinen scheint ein Konsens darüber zu bestehen, dass eine angemessene Vorbereitung vor Beginn des Studiums erhebliche positive Auswirkungen auf die spätere akademische Leistung internationaler Studierender hat. In welcher Form diese Studienvorbereitung ideal auf ein Studium an einer deutschen Hochschule vorbereiten und wie dies gewährleistet werden kann, ist dabei noch nicht umfassend geklärt.

Die vorliegende Überblicksstudie fasst die Ergebnisse einer Untersuchung zu Studienvorbereitung und -einstieg internationaler Studierender in Deutschland, die zwischen Mai 2019 und Januar 2020 durch den DAAD durchgeführt wurde, zusammen. Basierend auf einer Befragung von Hochschulvertretern und -vertreterinnen untersucht diese Publikation, wie deutsche Hochschulen internationale Studierende auf ein Studium in Deutschland vorbereiten.

Die vorliegende Publikation gelangte zu folgenden Ergebnissen:

\section{Maßnahmen}

Die Untersuchung zeigt, dass die überwiegende Mehrheit der Hochschulen studienvorbereitende Maßnahmen für internationale Studierende anbietet. Die meisten dieser Angebote zur Studienvorbereitung können als freiwillige und kostenlose Formate von den Studierenden wahrgenommen werden. Zum größten Teil bieten die Hochschulen studienvorbereitende Formate in Präsenz an. Darüber hinaus besteht ein relativ breites Angebot an rein digitalen Formaten oder integriertem Lernen (Blended Learning").

\section{Strukturen}

Bei den Zuständigkeiten hinsichtlich Konzeption, Umsetzung und Durchführung studienvorbereitender Maßnahmen sind zwischen den Institutionen bedeutende Unterschiede festzustellen. In den meisten Fällen werden die Maßnahmen von dem jeweiligen International Office beziehungsweise Akademischen Auslandsamt angeboten. Oft tragen auch die jeweiligen Fakultäten, Fachbereiche, Studienberatungen oder das Sprachenzentrum Verantwortung für die Maßnahmen. In einigen Fällen ist außerdem das Studiensekretariat der Hochschule involviert. Sofern ein Studienkolleg vorhanden ist, spielt dieses bei der Vorbereitung der Studienbewerberinnen und -bewerber eine zentrale Rolle.

\section{Praxisbeispiele}

Auf Basis der systematischen Analyse der Praxisbeispiele, die im Rahmen der Untersuchung identifiziert wurden, stellt diese Überblicksstudie einige Maßnahmen dar, die aus konzeptioneller Sicht als sinnvoll erscheinen. Es sei darauf hingewiesen, dass die Studie keine Aussagen bezüglich der Effektivität der identifizierten und dargestellten Maßnahmen treffen kann.

Abschließend thematisiert die Studie aktuelle Entwicklungen und liefert eine Prognose zu Modellen studienvorbereitender Maßnahmen, die zukünftig entwickelt und implementiert werden könnten. Hierbei werden sowohl digitale Maßnahmen als auch Präsenz-Studienvorbereitung, welche im Heimatland absolviert werden kann und von deutschen Hochschulen zur Verfügung gestellt wird, betrachtet. 

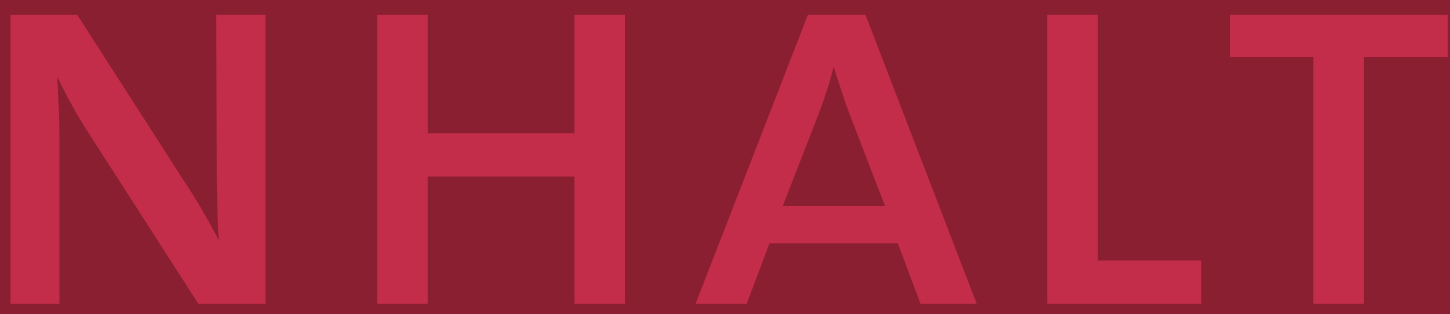


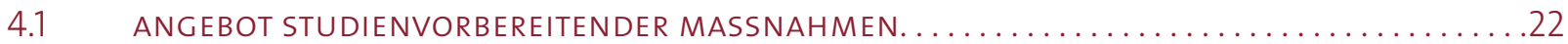

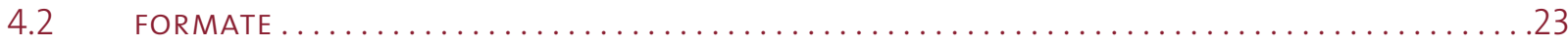

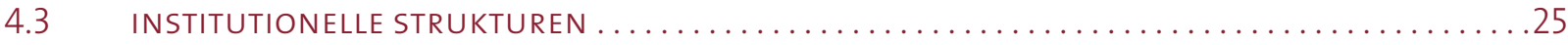

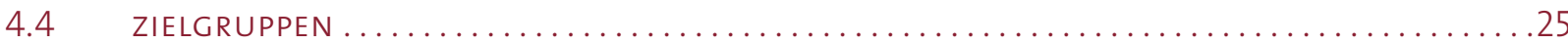

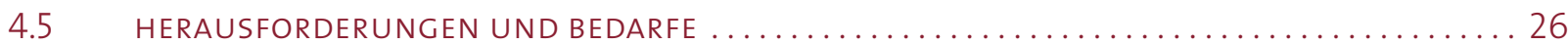

$5 \quad$ Praxisbeispiele an deutschen Hochschulen $\quad 30$

$6 \quad$ Fazit und Ausblick 38

$7 \quad$ Methodische Durchführung der Studie $\quad 40$

Literatur $\quad 42$

$\begin{array}{ll}\text { Anhang } & 44\end{array}$ 
ABBILDUNG VERZEICHNIS 
Abbildung 1

Abbildung 2

Abbildung 3

Abbildung 4

Abbildung 5

Abbildung 6

Abbildung 7

Abbildung 8
Antwortverhalten der eingeladenen Institutionen

21

Teilnehmende Institutionen nach Hochschulart

Angebot studienvorbereitender Maßnahmen

22

Art der angebotenen studienvorbereitenden Maßnahmen

22

Studienvorbereitende Angebote und Testverfahren, die Einfluss auf die Zulassung von Studienbewerberinnen und -bewerbern ohne Hochschulzugangsberechtigung haben

Stand der Digitalisierung in den Formaten nach Hochschulart

Zuständigkeit der studienvorbereitenden Maßnahmen

Zielgruppen der studienvorbereitenden Maßnahmen 
Einleitung 
Weltweit ist mit stabiler Tendenz ${ }^{1}$ eine Steigerung der internationalen Studierendenmobilität zu beobachten. In den letzten Jahrzehnten hat sich das Studium im Ausland zu einem angestrebten Bildungsweg für Studierende aus aller Welt entwickelt. Die Zahl der Studierenden, die an Hochschulen außerhalb ihres Heimatlandes eingeschrieben sind, ist in den letzten Jahren erheblich gestiegen (DAAD und DZHW, 2019). In diesem Kontext hat sich Deutschland als einer der weltweit führenden internationalen Studienstandorte positioniert. Das von Bund und Ländern vereinbarte Mobilitätsziel ausländischer Studierender ${ }^{2}$, dessen Erfüllung für 2020 angestrebt war, konnte so bereits 2017 erreicht werden. Deutschland steht hinter den Vereinigten Staaten, Großbritannien und Australien auf Platz 4 der Gastländer für internationale Studierende. Unter den nicht-englischsprachigen Ländern ist Deutschland damit das Land mit der höchsten Zahl internationaler Studierender in der Welt (DAAD und DZHW, 2019). Eine aktuelle Studie des British Council (British Council, 2019) vergleicht Rahmenbedingungen und Resultate der Internationalisierung der Hochschulbildung in europäischen Ländern, Amerika sowie Australien, China und Indien. Dem Bericht zufolge belegt Deutschland aufgrund exzellenter Internationalisierungsstrategien im Hochschulbereich, hoher internationaler Studierendenmobilität, transnationaler Bildung, internationaler Forschungskooperationen und nachhaltiger Entwicklung hinter den Niederlanden Platz 2 des Rankings.

Die deutsche Hochschullandschaft ist vielfältig und bietet zahlreiche Möglichkeiten für Studieninteressierte aus dem Ausland. Laut der Hochschulrektorenkonferenz (HRK) besteht Deutschlands Hochschulsystem aus 394 Hochschulen (121 Universitäten, 216 Fachhochschulen, 57 Kunst- und Musikhochschulen). 115 davon sind privat und 39 in kirchlicher Trägerschaft ${ }^{3}$. Einer Untersuchung des Centrums für Hochschulentwicklung (siehe Hachmeister und Grevers, 2019) zufolge bieten Hochschulen in Deutschland aktuell 20.123 Studiengänge (49\% Bachelor bzw. $51 \%$ Master) an. Die Sichtbarkeit des deutschen Hochschulsystems wird durch die Platzierung einiger deutscher Hochschulen unter den Top 200 Hochschulen in internationalen Rankings, wie den „OS World University Rankings“ oder dem „Times Higher Education World University Ran- king“, deutlich erhöht. Vielfältigkeit und internationale Reputation der deutschen Hochschulbildung gehen mit relativ geringen Studiengebühren und Lebenshaltungskosten einher. Abgesehen von einem Semesterbeitrag in Höhe von 40 bis 350 Euro, der sich aus Beiträgen für das Studierendenwerk (Mensen, Wohnheime und Beratungsstellen), die studentische Selbstverwaltung sowie die Nutzung von öffentlichen Verkehrsmitteln zusammensetzt, wird das Studium in Deutschland in den meisten Fällen vom Staat finanziert und ist deshalb für die Studierenden kostenlos ${ }^{4}$.

Morris-Lange (2019) nennt eine Reihe deutscher Organisationen, die für die Begleitung internationaler Studieninteressierter zur Verfügung stehen. Laut dem Autor beteiligen sich an der Gewinnung und Bindung internationaler Studierender zahlreiche Akteure, wie Hochschulen, deutsche Auslands- und Partnerschulen, deutsche Sprachkursanbieter im Ausland, der Deutsche Akademische Austauschdienst (DAAD), das Goethe-Institut, die Hochschulrektorenkonferenz (HRK), Studienkollegs im In- und Ausland, die Arbeits- und Servicestelle für ausländische Studienbewerbungen (uni-assist e.V.), die deutschen Auslandsvertretungen, das Deutsche Studierendenwerk, die Gesellschaft für Akademische Studienvorbereitung und Testentwicklung (g.a.s.t.), sowie die Kultusministerkonferenz (KMK).

Hoffmeyer-Zlotnik und Grote (2019) unterscheiden zwischen unterschiedlichen Maßnahmen zur Gewinnung internationaler Studierender in Deutschland. Ein Teil der Maßnahmen sind Initiativen, die gezielte Informationsangebote bereitstellen und Marketingmaßnahmen umsetzen ${ }^{5}$. Weiterhin thematisieren die Autoren die Einführung von englischsprachigen Studienangeboten als wichtigen Mechanismus zur Gewinnung internationaler Studierender. Seit der Einführung von Masterstudiengängen im Rahmen des Bologna-Prozesses hat sich die Zahl der englischsprachigen Studiengänge vervielfacht. Dementsprechend waren im Jahr $2019 \mathrm{im}$ HRK-Hochschulkompass 1.467 englischsprachige Studiengänge registriert (80\% davon Masterstudiengänge). Der letzte Aspekt, auf den Hoffmeyer-Zlotnik und Grote eingehen, sind Maßnahmen zur sprachlichen und fachlichen Vorbereitung auf das Studium. Die Autoren nennen unter „Studien-

$1 \quad$ Zum Zeitpunkt der Abfassung dieses Berichts kämpft die Welt gegen die Ausbreitung des Coronavirus (COVID-19-Pandemie). Aktuell ist noch nicht absehbar, welche langfristigen Auswirkungen auf die Entwicklung der internationalen Studierendenmobilität zu erwarten sind. Gegenwärtig erscheint eine Vielzahl an Analysen und Prognosen zum möglichen Corona-Effekt im Hochschulbereich. Einige Umfrageergebnisse zeigen, dass die Mehrzahl internationaler Studierender weiterhin an einem Auslandsstudium interessiert ist. Der DAAD verfolgt diese Entwicklung und gibt einen umfassenden Überblick zum aktuellen Stand der Forschung zu Folgen der Pandemie im Hochschulbereich: www.daad.de/corona-studien.

2 In Deutschland haben die Gemeinsame Wissenschaftskonferenz von Bund und Ländern (GWK) sowie der DAAD im Jahr 2013 das Ziel definiert, dass die Zahl der ausländischen Studierenden bis 2020 auf 350.000 steigen soll (GWK, 2013; DAAD \& DZHW, 2018).

Siehe: https://www.hrk.de/themen/hochschulsystem/statistik/

4 Eine Ausnahme stellen die Gebühren von 1.500 € pro Semester für Nicht-EU-/EWR-/Schweizer Studierende im Bundesland Baden-Württemberg dar. Ab dem Wintersemester 2017/18 wurden diese Studiengebühren für Bachelor, Master, Diplom und Staatsexamen eingeführt.

5 Unter anderem können hier erfolgreiche Initiativen wie das Marketingkonsortium GATE-Germany mit Kampagnen wie „Study in Germany - Land der Ideen" und „Research in Germany - Land der Ideen" genannt werden. 
kollegs und Studienvorbereitungskurse" Formate wie Studienkollegs, Vorstudium oder Propädeutika ${ }^{6}$ an Hochschulen, Vorkurse für bestimmte Studiengänge, Sprachkurse deutscher Hochschulen sowie das vom Goethe-Institut mit Partnern durchgeführte Programm „Studienbrücke“, welches eine Vorbereitung in den Herkunftsländern anbietet, hauptsächlich in Blended Learning-Formaten.

Während internationale Studierende das deutsche Hochschulsystem in vielen Aspekten bereichern, stellt die zunehmende Heterogenität der Herkunft der Studierenden die Hochschulen vor eine Reihe von praktischen Herausforderungen, um notwendige Unterstützungsleistungen zu gewährleisten. Schätzungen des Deutschen Zentrums für Hochschul- und Wissenschaftsforschung (DZHW) zufolge brechen $45 \%$ der in ternationalen Studierenden (versus $28 \%$ der deutschen Studierenden) im Bachelor bzw. 29\% (versus 19\%) im Master ihr Studium ab (Heublein und Schmelzer, 2018). Bislang liegen keine validen Daten und Erkenntnisse der Hochschulforschung zu den Gründen für die hohen Studienabbruchsraten vor. Aktuell beschäftigen sich daher zahlreiche Studien mit der Erforschung des Phänomens ${ }^{7}$. Einige Analysen weisen darauf hin, dass internationale Studierende aufgrund ihrer heterogenen Bildungshintergründe oft nicht über die notwendige studienbezogene Vorbereitung verfügen (Morris-Lange, 2017). Im Allgemeinen gibt es sowohl in der Literatur als auch in der Praxis einen Konsens darüber, dass eine angemessene Vorbereitung ${ }^{8}$ vor Beginn des Studiums erhebliche Auswirkungen auf die spätere Leistung und die Wahrscheinlichkeit des Erfolgs internationaler Studierender haben kann. Die Erhöhung des Studienerfolgs internationaler Studierender und des Gelingens des Karriereeinstiegs sind wichtige strategische Ziele für das BMBF und den DAAD mit Blick auf die demographische Entwicklung Deutschlands. Untersuchungen des Sachverständigenrats deutscher Stiftungen für Integration und Migration (SVR) zufolge möchten rund 7 von 10 internationalen Studierenden nach dem Studium in Deutschland bleiben und arbeiten (Morris-Lange et al., 2015; Morris-Lange und Brands, 2015; Lokhande, 2017).

In Deutschland gibt es eine Reihe unterschiedlicher studienvorbereitender Maßnahmen. Dies beginnt bei fakultativen Maßnahmen zur Orientierung am Hochschulort und reicht bis zum erfolgreichen Absolvieren des Studienkollegs und dem damit verbundenen Erwerb der Hochschulzugangsberechtigung (HZB). In einigen Bundesländern werden vermehrt alternative Wege ins Studium entwickelt: Statt der Feststellungsprüfung (FSP) werden der Test für Ausländische Studierende (TestAS), eigene Testverfahren oder Propädeutika für die Vorbereitung und Gewinnung von Bewerberinnen und Bewerbern genutzt. Das sich hieraus ergebende Bild ist unübersichtlich und nicht nur für internationale Bewerberinnen und Bewerber, sondern auch für Hochschulpraktikerinnen und -praktiker, die Forschung und die Politik verwirrend. Die vorliegende Studie soll Hinweise darauf liefern, wie die deutschen Hochschulen besser dabei unterstützt werden können, die für sie passenden Studierenden weltweit anzusprechen und für ein Studium in Deutschland zu gewinnen. Im Mittelpunkt dieser Publikation steht die Frage, wie genau deutsche Hochschulen internationale Studierende auf das Studium vorbereiten.

6 Ein Propädeutikum ist meist eine Vorbereitungsveranstaltung, die Wissen vor der Aufnahme eines Studiums vermitteln soll.

Siehe Falk et al. (2019); Wisniewski (2018) und Grüttner et al. (2018).

Allerdings stellt sich noch die Frage, was genau eine ideale (Studien-)Vorbereitung beinhaltet und wie diese zu gewährleisten ist. 


\section{Erstellung eines \\ Befragungskonzepts 9}

2.1 ERSTE PHASE: ANALYSE DER RECHTLICHEN SITUATION INTERNATIONALER STUDIERENDER BEZÜGLICH IHRER STUDIENVORBEREITUNG

2.2 ZWEITE PHASE: LITERATURRECHERCHE ZU STUDIENVORBEREITENDEN MASSNAHMEN AN DEUTSCHEN HOCHSCHULEN

2.3 DRITTE PHASE: ANALYSE DER IN DER DATENBANK „INTERNATIONAL PROGRAMMES IN GERMANY“ FÜR DEUTSCHLAND AUFGELISTETEN ANGEBOTE SOWIE VON ÄHNLICHEN ANGEBOTEN IN AUSGEWÄHLTEN EUROPÄISCHEN LÄNDERN

2.4 VIERTE PHASE: DEDUKTIVE ERGÄNZUNGEN BASIEREND AUF BEOBACHTETEN PRAXISBEISPIELEN UND DAAD-EXPERTISE

2.5 FÜNFTE PHASE: ERGÄNZENDE BEFRAGUNG VON VERTRETERINNEN UND VERTRETERN EINZELNER INSTITUTIONEN 
Angesichts der explorativen Natur der Untersuchung wurde ein analytisches 5-Phasen-Modell entwickelt. In einer ersten Phase wurde die Thematik aus einer Makro-Perspektive untersucht, um die rechtlichen sowie gesellschaftlichen Rahmenbedingungen der Studienvorbereitung internationaler Studierender in Deutschland abzubilden. Im Anschluss an die Identifikation von Grenzen und Möglichkeiten der rechtlichen Situation wurde eine Literaturrecherche zu studienvorbereitenden Maßnahmen an deutschen Hochschulen durchgeführt. Ziel dieses Schrittes war die Entwicklung eines Kategoriensystems mit den geläufigen Begrifflichkeiten und die Erfassung bestehender Angebote. Die dritte Phase diente der Analyse von Angeboten in der Datenbank „International Programmes in Germany“ sowie ähnlichen Programmen ausgewählter europäischer Länder, um weitere Kategorien zu identifizieren. Basierend auf Praxisbeispielen und der Expertise des DAAD in diesem Themenbereich wurde außerdem eine allgemeine Arbeitsdefinition von studienvorbereitenden Maßnahmen für internationale Studierende vorgeschlagen und wurden die Themen der Befragung präzisiert. In der letzten Phase wurden schließlich Befragungen und Interviews mit Ansprechpartnerinnen und -partnern für studienvorbereitende Maßnahmen durchgeführt. Dieser Dialog diente der Erstellung von Praxisbeispielen und der Ergänzung der Ergebnisse. Im Folgenden werden die einzelnen Phasen näher erläutert, um die Entwicklung der Untersuchung nachvollziehbar darzustellen.

\subsection{ERSTE PHASE: ANALYSE DER RECHTLICHEN SITUATION INTERNATIONALER STUDIERENDER BEZÜGLICH IHRER STUDIENVORBEREITUNG}

\begin{abstract}
Aus der Recherche zu diesem Thema ergibt sich ein komplexes Bild von Regelungen und Akteuren, die internationale Studienbewerberinnen und -bewerber bei Hochschulzugang, -zulassung und ihrer Bewerbung ${ }^{10}$ für ein Studium in Deutschland berücksichtigen müssen. In Deutschland spielen gesetzliche Regelungen eine entscheidende Rolle beim Erwerb der Hochschulzugangsberechtigung. Der Zugang zum Studium an deutschen Hochschulen ist grundsätzlich auch für Studieninteressierte mit sowohl ausländischer Staatsangehörigkeit als auch ausländischer Hochschulzugangsberechtigung möglich.
\end{abstract}

EU-Bürger, Bürger der EWR-Staaten und der Schweiz müssen keinen Aufenthaltstitel beantragen, um ein Studium in Deutschland aufnehmen zu dürfen. Für internationale Studierende aus Nicht-EU-Ländern ist das Aufenthaltsrecht durch $\$ 16$ des Gesetzes über den Aufenthalt, die Erwerbstätigkeit und die Integration von Ausländern im Bundesgebiet (Aufenthaltsgesetz - AufenthG) „§16 Studium“ geregelt. Gemäß dem genannten Gesetz kann eine Aufenthaltserlaubnis auch für die Teilnahme an studienvorbereitenden Maßnahmen erteilt werden. Hierzu zählen: Studienvorbereitende Sprachkurse, der Besuch eines Studienkollegs oder einer vergleichbaren Einrichtung und das Absolvieren eines studienvorbereitenden Praktikums. Im Fall von Sprachkursen sowie Studienkollegs o.ä. kann die Aufenthaltserlaubnis auch ohne die Zulassung zu einer Hochschule bewilligt werden ${ }^{11}$. Angehörige der betroffenen Staaten müssen vor oder (je nach Heimatland) nach Einreise in Deutschland ein Studentenvisum oder ein Visum für die Studienbewerbung bzw. -vorbereitung beantragen ${ }^{12}$.

\subsection{ZWEITE PHASE: LITERATURRECHERCHE ZU STUDIENVORBEREITENDEN MASSNAHMEN AN DEUTSCHEN HOCHSCHULEN}

Vorangehend wurden studienvorbereitende Maßnahmen wie Sprachkurse, der Besuch von Studienkollegs oder vergleichbaren Einrichtungen sowie das Absolvieren eines Praktikums genannt. Im Folgenden sollen nun die Ergebnisse einer systematischen Literaturre- cherche zu zielgruppenunabhängigen Maßnahmen für die Studienvorbereitung an deutschen Hochschulen vorgestellt werden. Insgesamt wurden hierfür 75 Publikationen identifiziert und thematisch analysiert. Hierunter waren Quellen wie Buchbeiträge, Sammel-

9 Im Rahmen dieser Publikation werden nur Maßnahmen analysiert, die für internationale Studierende nach ihrer Ankunft in Deutschland angeboten werden. Nicht eingeschlossen wurden Maßnahmen, die im Ausland angeboten werden (z.B. Studienkollegs im Ausland oder das Programm „Studienbrücke"), besondere Modelle wie der Status als Gasthörer oder ein Schnupperstudium sowie Initiativen zur Personalisierung des Hochschuleinstiegs durch Digitalisierung (z.B. Projekt „IMPact Digital - Internationalisierung deutscher Hochschulen“).

10 Je nach erworbener Hochschulzugangsberechtigung und Zulassungsbeschränkung der ausgewählten Hochschule erwarten die internationalen Studienbewerberinnen und -bewerber unterschiedliche Anforderungen an ein Studium in Deutschland.

11 Der Recherche für diese Studie zufolge ist festzuhalten, dass in den einzelnen Bundesländern unterschiedlich mit den Regelungen aus dem Aufenthaltsgesetz umgegangen wird. Siehe Borgwardt et al. (2015) für eine Analyse der verschiedenen Regelungen des Hochschulzugangs für Flüchtlinge in den einzelnen Bundesländern.

12 Für Visumsanträge wird seit dem 1. September 2019 ein Finanzierungsnachweis von $10.236 €$ pro Jahr bzw. $853 €$ pro Monat für Staatsangehörige, die ein Visum bzw. einen Aufenthaltstitel für ein Studium in Deutschland benötigen, verlangt. 
bände, Forschungsberichte, Dokumentationen von Tagungen, Monographien, Handbücher, Positionspapiere, wissenschaftliche Publikationen, Evaluationen, Dissertationen und Arbeitspapiere. Basierend auf diesen wurde ein Kategoriensystem für Begrifflichkeiten entwickelt, die wiederkehrend in den Quellen Verwendung fanden. Anschließend wurde dieses in Themencluster unterteilt:

Kategoriensystem nach der Literaturrecherche

- Online-Kurse, Blended Learning

- Studienberatung

- Berufsorientierung, Studienorientierung

- Tutorenprogramme, Mentorenprogramme

- Online-Self-Assessments, Self-Assessments

- Software und Apps im Studium, wissenschaftliches Arbeiten, Studienkompetenzen

- Interkulturelle Sensibilisierung

- Förderung und Überprüfung von Fachkenntnissen, Studienkollegs

- Studienvorbereitende Sprachkurse
Im Rahmen der Recherche wurde festgestellt, dass bisher wenige Publikationen über studienvorbereitende Maßnahmen für internationale Studierende vorliegen. Darüber hinaus fiel auf, dass die meisten Publikationen Initiativen einzelner Hochschulen oder sogar Fachbereiche vorstellen und diskutieren. Ein konklusiver, aussagekräftiger Überblick zu Maßnahmen dieser Art liegt folglich noch nicht vor. Eine Ausnahme stellt die Publikation „Orientierung und Unterstützung zum Studieneingang: Umsetzungsstand an deutschen Hochschulen" von Hill und Key (2019) dar. Allerdings erwähnen die Autoren in der Publikation lediglich, es gäbe „an vielen Hochschulen gesonderte Veranstaltungen und Angebote für ausländische Studierende oder Studierende mit Kindern" (Hill und Key, 2019 S. 18), ohne weitere Erläuterungen zu den Maßnahmen zu geben.

2.3 DRITTE PHASE: ANALYSE DER IN DER DATENBANK „INTERNATIONAL PROGRAMMES IN GERMANY“ FÜR DEUTSCHLAND AUFGELISTETEN ANGEBOTE SOWIE VON ÄHNLICHEN ANGEBOTEN IN AUSGEWÄHLTEN EUROPÄISCHEN LÄNDERN

Um die im vorherigen Schritt erarbeiteten Inhaltscluster zu ergänzen, erfolgte in Phase 3 die Analyse der in der Datenbank „International Programmes in Germany" gelisteten Angebote sowie ähnlicher Angebote ausgewählter europäischer Länder. Diese Analyse hat gezeigt, wie unterschiedlich die Modalitäten und In- halte studienvorbereitender Maßnahmen aufgebaut sind. Zusätzlich wurden relevante Merkmale für die Untersuchung identifiziert, wie die Unterscheidung zwischen freiwilligen und obligatorischen sowie kostenlosen und kostenpflichtigen Maßnahmen.

\subsection{VIERTE PHASE: DEDUKTIVE ERGÄNZUNGEN BASIEREND AUF BEOBACHTETEN PRAXISBEISPIELEN UND DAAD-EXPERTISE}

Der letzte konzeptionelle Schritt der Analyse war die Diskussion sowohl des bisherigen Kategoriensystems als auch des vorläufigen Entwurfs eines Fragebogens unter Berücksichtigung von bekannten Praxisbeispielen und dem Expertenwissen des DAAD. Nach diesem Schritt wurde die folgende Definition von "Maßnah- men zur Studienvorbereitung für internationale Studierende" festgelegt:

In Anlehnung an Schellhorn et al. (2019) werden unter "Maßnahmen zur Studienvorbereitung" Angebote verstanden, welche von Hochschulen selbst oder von 
Partnerorganisationen mit dem Ziel initiiert werden, den Studieneinstieg zu erleichtern. Sie begleiten die internationalen Studierenden bzw. Studieninteressierten vor Beginn ihres Studiums in Deutschland und zielen darauf $a b$, wichtige Studienbereiche und teilweise fachliche Inhalte zu vermitteln. Auf diese Weise sollen curriculare Lücken ausgeglichen und Schlüsselkompetenzen erworben werden, die für das Studium in Deutschland notwendig sind. Darüber hinaus gibt es eine Reihe von Maßnahmen, deren erfolgreiches Absolvieren sich auf den Hochschulzugang internationaler Studierender auswirken kann. ${ }^{13}$

\subsection{FÜNFTE PHASE: ERGÄNZENDE BEFRAGUNG VON VERTRETERINNEN UND VERTRETERN EINZELNER INSTITUTIONEN}

In der letzten Phase des analytischen 5-Phasen-Modells wurden individuelle Befragungen und Interviews mit Ansprechpartnerinnen und -partnern für studien- vorbereitende Maßnahmen durchgeführt. Dieser Dialog diente dem Zusammenstellen von Praxisbeispielen und der qualitativen Ergänzung der Ergebnisse.

13 Ausgehend von dieser Studie wurde ein Kategoriensystem für die Befragung entwickelt, welches in Kapitel 7 zu finden ist. 
Fragestellungen 
In dieser Publikation sollen auf Basis der beschriebenen Analysen insbesondere folgende Fragen behandelt werden:

- Werden studienvorbereitende Maßnahmen für internationale Studierende an deutschen Hochschulen bzw. Partnerorganisationen angeboten? Welche sind die am häufigsten angebotenen Maßnahmen?

- Bieten Hochschulen bzw. Partnerorganisationen studienvorbereitende Maßnahmen an, welche die Studieninteressierten nutzen können, die aber keine Relevanz für ihren Zugang zum Studium haben? Wo liegen Schwerpunkte?

- Werden studienvorbereitende Maßnahmen bzw. Testverfahren angeboten, deren erfolgreiches Absolvieren bei der Zulassung von Studienbewerberinnen und Studienbewerbern, insbesondere ohne HZB, berücksichtigt wird? Wo liegen Schwerpunkte?
- Ist die Teilnahme an den studienvorbereitenden Maßnahmen freiwillig oder obligatorisch?

- Sind die studienvorbereitenden Maßnahmen kostenlos oder kostenpflichtig?

- Welche Formate (Digital, Blended Learning, Präsenz) werden an den Institutionen angeboten?

- Wer ist an den Hochschulen für die studienvorbereitenden Maßnahmen zuständig? Sind besondere Strukturen festzustellen?

- Konzentrieren sich die studienvorbereitenden Maßnahmen auf eine bestimmte Zielgruppe? Wo liegen Schwerpunkte?

- Existieren Herausforderungen oder Hindernisse bzw. Bedarfe im Bereich der studienvorbereitenden Maßnahmen? 


\section{Diskussion aus- gewählter Ergebnisse ${ }^{14}$}

4.1 ANGEBOT STUDIENVORBEREITENDER MASSNAHMEN

4.2 FORMATE

4.3 INSTITUTIONELLE STRUKTUREN

4.4 ZIELGRUPPEN

4.5 HERAUSFORDERUNGEN UND BEDARFE 
Die vorliegende Überblicksstudie fasst die Ergebnisse einer Untersuchung zu Studienvorbereitung und -einstieg internationaler Studierender in Deutschland, die zwischen Mai 2019 und Januar 2020 durch den DAAD durchgeführt wurde, zusammen. Basierend auf einer Befragung von Hochschulvertretern und -vertreterinnen untersucht diese Publikation, wie deutsche Hochschulen internationale Studierende auf ein Studium in Deutschland vorbereiten. Insgesamt wurden 210 deut- sche Hochschulen zur Umfrage eingeladen, von denen 112 (53\%) den Fragebogen beantworteten. Die durch die Umfrage generierten Daten können nicht als statistische Erhebung betrachtet werden. Die Datengrundlage ist heterogen und beruht zum großen Teil auf Schätzungen oder dem Kenntnisstand der jeweiligen Ansprechpartnerinnen und -partner. Die Effektivität oder Wirkung der angebotenen studienvorbereitenden Maßnahmen kann an dieser Stelle nicht untersucht werden.

Abbildung 1

Antwortverhalten der eingeladenen Institutionen, Angaben in absoluten Zahlen

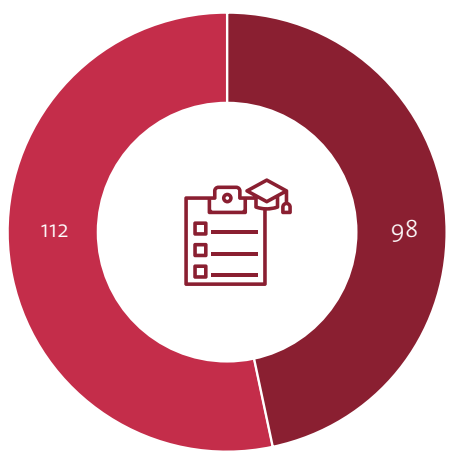

- Nicht teilgenommen

- Teilgenommen

Jeweils $47 \%$ der an der Umfrage teilnehmenden Hochschulen waren Universitäten und Fachhochschulen bzw. Hochschulen für angewandte Wissenschaften; auf die privaten Hochschulen entfielen knapp $6 \%$. Die geringe Teilnahme von privaten Hochschulen kann teil- weise damit erklärt werden, dass ausschließlich HRKMitgliedshochschulen befragt wurden, unter denen private Hochschulen unterrepräsentiert sind. Aussagen in Bezug auf die Unterschiede bezüglich der Beteiligung von Hochschultypen sind daher schwer zu treffen.

Abbildung 2

Teilnehmende Institutionen nach Hochschulart, Angaben in Prozent

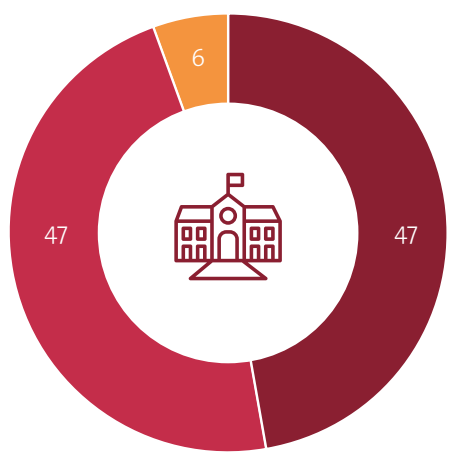

- Universitäten

- Fachhochschulen/Hochschulen für angewandte Wissenschaften

- Private Hochschulen 


\section{ANGEBOT STUDIENVORBEREITENDER MASSNAHMEN}

Die Umfrage zeigt, dass die überwiegende Mehrheit der teilnehmenden Institutionen (91\%) studienvorbereitende Maßnahmen anbietet.

Abbildung 3

Angebot studienvorbereitender Maßnahmen, Angaben in Prozent

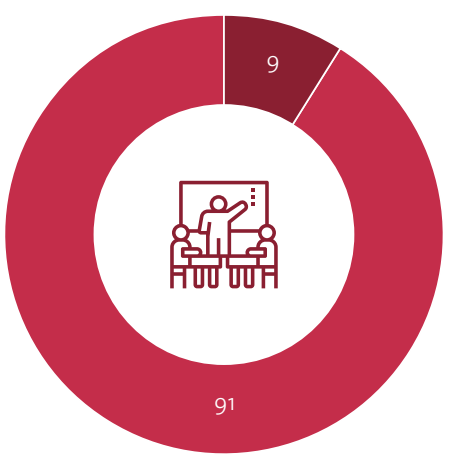

- studienvorbereitende Maßnahmen werden nicht angeboten

- studienvorbereitende Maßnahmen werden angeboten

Es wurden sowohl Add-on-Angebote, die Studierende bei Bedarf nutzen können, als auch Maßnahmen, deren erfolgreiches Absolvieren sich auf den Hochschulzugang auswirkt, identifiziert. Betrachtet man die angebotenen studienvorbereitenden Maßnahmen näher, zeigt sich eine ganze Bandbreite an Angeboten. An erster Stelle stehen Angebote zur Studienorientierung, darunter fallen Online-Self-Assessments (insgesamt 90 Hochschulen bieten dies an). Danach folgen studienvorbereitende Sprachkurse (an 77 Hochschulen), sowie Mentoring- bzw. Tutorenprogramme (an 68 Hochschulen). 63 der Hochschulen bieten die Förderung und Überprüfung von Fachkenntnissen (z.B. Mathematik, Deutsch, Physik, Programmieren) an. Angebote zur interkulturellen Sensibilisierung im Sinne der deutschen (Wissenschafts-) Kultur werden von 50 Hochschulen angeboten. 46 Hochschulen bieten studienvorbereitende Maßnah- men in wissenschaftlichem Schreiben an. An fast ebenso vielen Hochschulen (42) können vor Studienbeginn Kompetenzen wie Selbstständigkeit, Zeitmanagement oder die Vorbereitung auf Sprechstunden- und Prüfungsgespräche verbessert werden. Je 24 Hochschulen stellen Studienkollegs, wie auch vorbereitende Kurse für die Nutzung von Software und Apps im Studium bereit. Vereinzelt werden (Vor-)Praktika (18) sowie Kurse für die Vorbereitung auf Eingangsprüfungen (16) angeboten. 25 Hochschulen gaben an, weitere Angebote bereitzustellen, die im Auswahlmenü nicht aufgeführt waren. Es handelt sich hierbei um sozio-kulturelle Angebote (4), Orientierungsangebote zum Semesterstart (3) und studienvorbereitende Angebote speziell für Geflüchtete (2). Zusätzlich existieren vorbereitende Maßnahmen, welche auf die Zugangsprüfungen (2) vorbereiten sowie fachliche Inhalte (2) vermitteln.

\section{Abbildung 4}

Art der angebotenen studienvorbereitenden Maßnahmen, Angaben in absoluten Zahlen

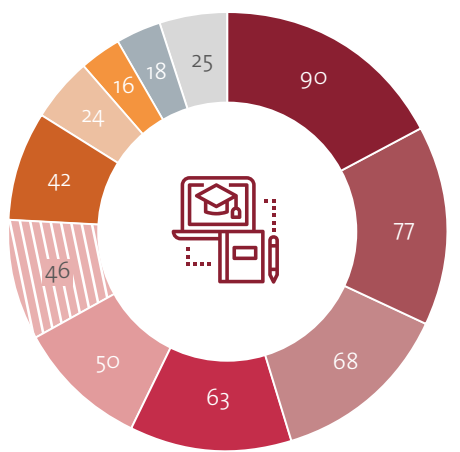

- Studienorientierung (Online-Self-Assessments, Studienberatung, Studienwahl)

- Studienvorbereitende Sprachkurse

- Mentoring-bzw. Tutorenprogramme

- Förderung und Überprüfung von Fachkenntnissen (z.B. Mathematik, Deutsch, Physik, Programmierung)

- Interkulturelle Sensibilisierung im Sinne der deutschen (Wissenschafts-)kultur werden

II Wissenschaftliches Arbeiten

- Studienkompetenzen (Selbstständigkeit, Zeitmanagement oder die Vorbereitung auf Sprechstunden- und Prüfungsgespräche)

- Software und Apps im Studium

- Vorbereitung auf Eingangsprüfungen

- (Vor-)Praktika

Sonstiges 
Maßnahmen für die Zulassung von

Studienbewerberinnen und -bewerbern

ohne Hochschulzugangsberechtigung

Im Kontext der Zulassungsverfahren internationaler Studierender müssen Hochschulen verschiedenste ausländische Bildungsabschlüsse bewerten und Entscheidungen über die Äquivalenz zu deutschen Hochschulzugangsberechtigungen treffen ${ }^{15}$. In den $50 e r$ Jahren wurde die Zentralstelle für ausländisches Bildungswesen ( $Z A B)$ etabliert, welche Empfehlungen zur Bewertung ausländischer Hochschulzugangsqualifikationen herausgibt. Diese Empfehlungen dienen der Orientierung sowohl seitens der Hochschulen als auch der Studieninteressierten. In der Regel wird für ausländische Studienbewerberinnen und -bewerber zwischen direkter Hochschulzugangsberechtigung und nicht direkter Hochschulzugangsberechtigung unterschieden. Laut der aktuellen „Rahmenordnung für den Hochschulzugang mit ausländischen Bildungsnachweisen, für die Ausbildung an den Studienkollegs und für die Feststellungsprüfung" besteht eine direkte Hochschulzugangsberechtigung für EU-Bürger, Angehörige von Staaten des Europäischen Wirtschaftsraumes sowie Inhaber einer ausländischen Hochschulzugangsberechtigung, welche gemäß den Bewertungsvorschlägen der Kultusministerkonferenz der deutschen Hochschulzugangsberechtigung gleichgestellt ist. Es gibt jedoch weitere Möglichkeiten des Zugangs zum Studium in Deutschland unter bestimmten Bedingungen, wie das International Baccalaureate Diploma (IB-Diploma) oder das General Certificate of Education (GCE) ${ }^{16}$. Zusätzlich müssen erforderliche Kenntnisse der deutschen bzw. anderer Sprachen ${ }^{17}$ nachgewiesen werden. Liegt durch den Schulabschluss keine direkte Hochschulzugangsberechtigung (HZB) vor, kann diese auf verschiedene Arten erlangt werden ${ }^{18}$.

Dank der Struktur des Fragebogens können Aussagen über die Rolle von eben solchen studienvorbereitenden Maßnahmen für internationale Studierende getroffen werden. Es zeigte sich, dass Angebote und Testverfahren, welche Einfluss auf die Zulassung von Studienbewerberinnen und -bewerbern ohne Hochschulzugangsberechtigung haben, vielfältig gestaltet sind. Am stärksten werden von Hochschulen für diese Zwecke Studienkollegs genutzt (31\%).
Studieninteressierten, deren Zeugnisse die Kriterien für den Hochschulzugang nicht erfüllen, stehen sogenannte Studienkollegs zur Verfügung. Studienkollegs sind Bildungseinrichtungen, in denen ausländische Studienbewerberinnen und -bewerber ohne direkte Hochschulzugangsberechtigung auf ein Studium an einer deutschen Hochschule vorbereitet werden. Das Kompetenzprofil der Studienkollegs sieht vor, eine Vermittlung von fundiertem Fachwissen sowie von allgemeinen, sprachlichen und fachlichen Kompetenzen zu gewährleisten, die für einen erfolgreichen Einstieg ins Studium grundlegend erforderlich sind. Der Weg an Studienkollegs kann je nach Bundesland und Hochschule sehr unterschiedlich verlaufen, wie die Tabelle in Anhang 1 zeigt. Die Bewerbung erfolgt teilweise direkt über das Studienkolleg, teilweise über die Hochschulen, in manchen Fällen ist auch die Arbeits- und Servicestelle für ausländische Studienbewerbungen (uni-assist e.V.) zuständig. Staatliche Studienkollegs sind häufig Teil einer Hochschule und nur für Bewerber an dieser oder bestimmten Hochschulen, meist im selben Bundesland, zugänglich. Die Aufnahme an privaten Kollegs ist dagegen größtenteils nicht an die Bewerbung bei einer bestimmten Hochschule gebunden. Weitere Unterschiede zwischen staatlichen und privaten Studienkollegs bestehen zudem hinsichtlich der Kosten; in der Regel sind in staatlichen Einrichtungen die regulären Semesteroder Verwaltungsbeiträge zu entrichten, während die Vorbereitung an privaten Studienkollegs mit deutlich höheren Gebühren verbunden ist. Zur Studienvorbereitung werden an den Studienkollegs Schwerpunktkurse angeboten, die fachlich relevante Inhalte, Fachthemen sowie disziplinäre Fragestellungen abdecken sollen ${ }^{19}$. Dabei ist zu unterscheiden zwischen Kursen für Universitäten und Kursen für Fachhochschulen; letztere erlauben nur die Aufnahme eines Studiums an diesem Hochschultyp.

Nach der Teilnahme an den Vorbereitungskursen eines in der Regel einjährigen Studienkollegs kann eine sogenannte Feststellungsprüfung abgelegt werden. Staatliche und staatlich anerkannte Studienkollegs sind dazu berechtigt, diese Prüfung selbst durchzuführen, Besucher von staatlich genehmigten Kollegs müssen diese an anderen Einrichtungen ablegen. Obwohl die Feststellungsprüfung grundsätzlich fachgebunden ist, kann sie durch Ergänzungsprüfungen auf weitere Fächer ausgeweitet werden. Die Feststellungsprüfung gilt als Hochschulzugangsberechtigung und ermöglicht es Studien-

15 Für eine Diskussion zum Umrechnungsverfahren ausländischer Sekundarabschlussnoten siehe Hell (2007) und Loy et al. (2009)

16 Das IB-Diploma und das General Certificate of Education (GCE) sind Schulabschlusse, die weltweit an vielen Schulen erworben werden können. Unter gewissen Bedingungen können diese als direkte, fachgebundene Hochschulzugangsberechtigung akzeptiert werden.

17 Für ein grundständiges Studium in Deutschland müssen internationale Studierende in der Regel fortgeschrittene Kenntnisse (etwa C1-Niveau) der deutschen Sprache nachweisen. Das geforderte Sprachniveau kann sich zwischen einzelnen Hochschulen und Studiengängen jedoch unterscheiden. Zusätzlich können Nachweise für andere Sprachen gefordert werden.

18 Im Rahmen dieser Studie wird weder auf Studienzeiten im Heimatland eingegangen, welche angerechnet werden können, noch auf spezielle alternative Hochschulzugänge.

19 Einige Beispiele sind: „M-Kurs“ für medizinische, biologische und pharmazeutische Studiengänge, „T-Kurs“ für mathematisch-naturwissenschaftliche oder technische Studiengänge, ,W-Kurs“ für wirtschafts- und sozialwissenschaftliche Studiengänge an Universitätskollegs, ,TI-Kurs“ für technische und ingenieurwissenschaftliche Studiengänge, „WW-Kurs“ für wirtschaftswissenschaftliche Studiengänge und „SW-Kurs“ für sozialwissenschaftliche und gesellschaftswissenschaftliche Studiengänge an Fachhochschulkollegs. 
interessierten, ihr Bewerbungsverfahren abzuschließen und ein Studium in Deutschland aufzunehmen. In allen Bundesländern können Studienkollegs das Ablegen einer externen Feststellungsprüfung ohne vorherigen Besuch der Vorbereitungskurse erlauben. Diese können teilweise bei zuständigen Stellen eines Landesministeriums abgelegt werden (z.B. in Nordrhein-Westfalen) ${ }^{20}$.

Als zweitstärkstes Verfahren zeigte sich mit 21\% die Nutzung des TestAS zur Feststellung der Studierfähigkeit. Der TestAS (Test for Academic Studies) ist ein zentraler, standardisierter Studierfähigkeitstest für ausländische Studierende, der kognitive Fähigkeiten, welche für das Studium in Deutschland von Bedeutung sind, überprüft. Er kann in deutscher oder englischer Sprache an lizenzierten Testzentren weltweit abgelegt werden. Der TestAS besteht aus einem Kerntest (Quantitative Probleme lösen, Beziehungen erschließen, Muster ergänzen und Zahlenreihen fortsetzen) und je nach Studienwunschvier studienfeldspezifischen Testmodulen (Geistes-, Kultur- und Gesellschaftswissenschaften ${ }^{21}$, Ingenieurwissenschaften ${ }^{22}$, Mathematik, Informatik und Naturwissenschaften ${ }^{23}$ und Wirtschaftswissenschaften $\left.^{24}\right)$. Teilnehmende können eines der vier Module auswählen.

Vergleichsweise weniger oft nutzen die befragten Hochschulen eigene Eingangstests (11\%). Im Rahmen von DAAD-Veranstaltungen lässt sich beobachten, dass immer mehr deutsche Hochschulen spezielle Eignungstests für internationale Studienbewerber einsetzen.
Manche dieser Tests sind Bestandteil des Auswahlverfahrens der Hochschule. Beispielsweise können folgende Bereiche identifiziert werden: mathematische Kenntnisse, logisches Schlussfolgern, Allgemeinbildung sowie Studienmotivation und Leistungsbereitschaft.

Eine propädeutische Vorbereitungsphase nutzen laut Angaben im Fragebogen 19\% der teilnehmenden Hochschulen $^{25}$. Die Analyse von Angeboten dieser Art zeigt, dass in manchen Bundesländern ein Propädeutikum als Ersatz des Studienkollegs absolviert werden kann. In anderen Fällen werden sie dagegen lediglich fakultativ angeboten. Diese Programme bereiten Studienbewerberinnen und -bewerber sprachlich und fachlich ganz gezielt auf einen gewünschten Studiengang oder Fachbereich vor. Häufig bestehen sie aus einer Einführung in das deutsche Studiensystem und in wissenschaftliche Arbeitstechniken und variieren in ihrer Länge zwischen wenigen Wochen und zwei vollen Semestern.

Unter Sonstiges (mit 18\%) gaben die befragten Hochschulen vor allem Vorbereitungskurse für die Deutsche Sprachprüfung für den Hochschulzugang (DSH) sowie eine Abnahme derselben an. Ebenfalls wurde als sprachlicher Zugang das Bestehen des Test Deutsch als Fremdsprache (TestDaF) beziehungsweise eines TOEFLTests angegeben. Auch wurde die Option eines Probeoder Vorbereitungsstudiums genannt. In einigen Fällen kommt der TestAS in Kombination mit anderen Vorbereitungsformaten zum Einsatz.

\section{Abbildung 5}

Studienvorbereitende Angebote und Testverfahren, die Einfluss auf die Zulassung von Studienbewerberinnen und -bewerbern ohne Hochschulzugangsberechtigung haben, Angaben in Prozent

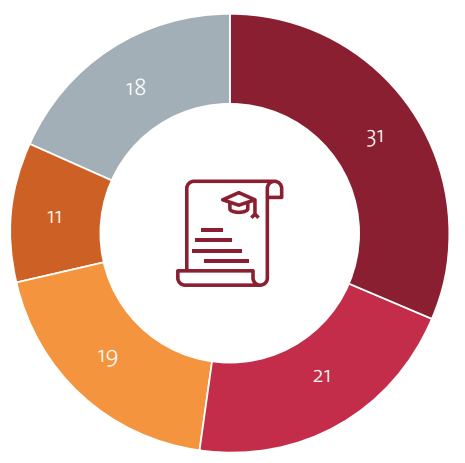

\section{- Studienkolleg}

- Nutzung des TestAS

- Propädeutische Vorbereitungsphase (z.B. Preparatory Semester, Pre-Study Programme)

\section{- Eigene Eingangstests}

- Sonstiges

In NRW sind die Bezirksregierungen Köln und Düsseldorf für die externe Feststellungsprüfung zuständig. Köln übernimmt dabei die zentrale Regelung der Feststellungsprüfung und Düsseldorf ist für die formale Überprüfung der Bewerbungsunterlagen zuständig. NRW scheint jedoch das einzige Bundesland zu sein, in dem ein Landesministerium die Abnahme der externen Feststellungsprüfung durchführt.

21 Aufgabengruppen:Texte verstehen und interpretieren, Repräsentationssysteme flexibel nutzen und Sprachstrukturen erkennen.

22 Aufgabengruppen:Technische Sachverhalte formalisieren, Ansichten erschließen und technische Zusammenhänge analysieren.

23 Aufgabengruppen: Naturwissenschaftliche Sachverhalte analysieren und Formale Darstellungen verstehen.

24 Aufgabengruppen: Wirtschaftliche Zusammenhänge analysieren und Prozesse analysieren.

25 In der Datenbank des DAAD finden sich 27 Programme unter den Begriffen: Foundation Year/ Programme, Prep Programme, Bridge Year, Studienstart, Pre-Study oder Freshman Programme. 
Bei der Einteilung der angebotenen Maßnahmen wurden die Art der Teilnahme sowie die Art der Finanzierung als Kategorien berücksichtigt. Es zeigte sich, dass die meisten Angebote zur Studienvorbereitung als überwiegend freiwillige und kostenlose Formate ${ }^{26}$ von den Studierenden wahrgenommen werden können. Zu diesen Formaten zählen Angebote zur Studienorientierung, der Förderung und Überprüfung von Fachkenntnissen, für den Erwerb von Studienkompetenzen, Mentoring- bzw. Tutorenprogramme, Kurse zu Software und Apps im Studium, zur kulturellen Sensibilisierung sowie zum wissenschaftlichen Arbeiten. In die Kategorie eines

\section{FORMATE}

Etwas mehr als die Hälfte der studienvorbereitenden Formate werden in Präsenz angeboten (54\%). Insgesamt obligatorischen und kostenlosen Angebotes fielen überwiegend Studienkollegs. Als freiwilliges und größtenteils kostenloses Angebot wurde die Vorbereitung auf Eingangsprüfungen genannt. Die Durchführung eines (Vor-)Praktikums wurde vorrangig als kostenloses und obligatorisches Angebot angegeben. Ein differenziertes Bild ergibt sich bei den studienvorbereitenden Sprachkursen. Hier zeigt sich, dass Angebote hauptsächlich auf freiwilliger Basis (72), teilweise aber auch obligatorisch (13) konzipiert sind. Darüber hinaus handelt es sich um sowohl kostenpflichtige Angebote (49) als auch um zahlreiche kostenlose Sprachangebote (49).

$21 \%$ der Formate werden rein digital angeboten, $25 \%$ in einer Form des integrierten Lernens (Blended Learning).

Abbildung 6

Stand der Digitalisierung in den Formaten nach Hochschulart, Angaben in Prozent

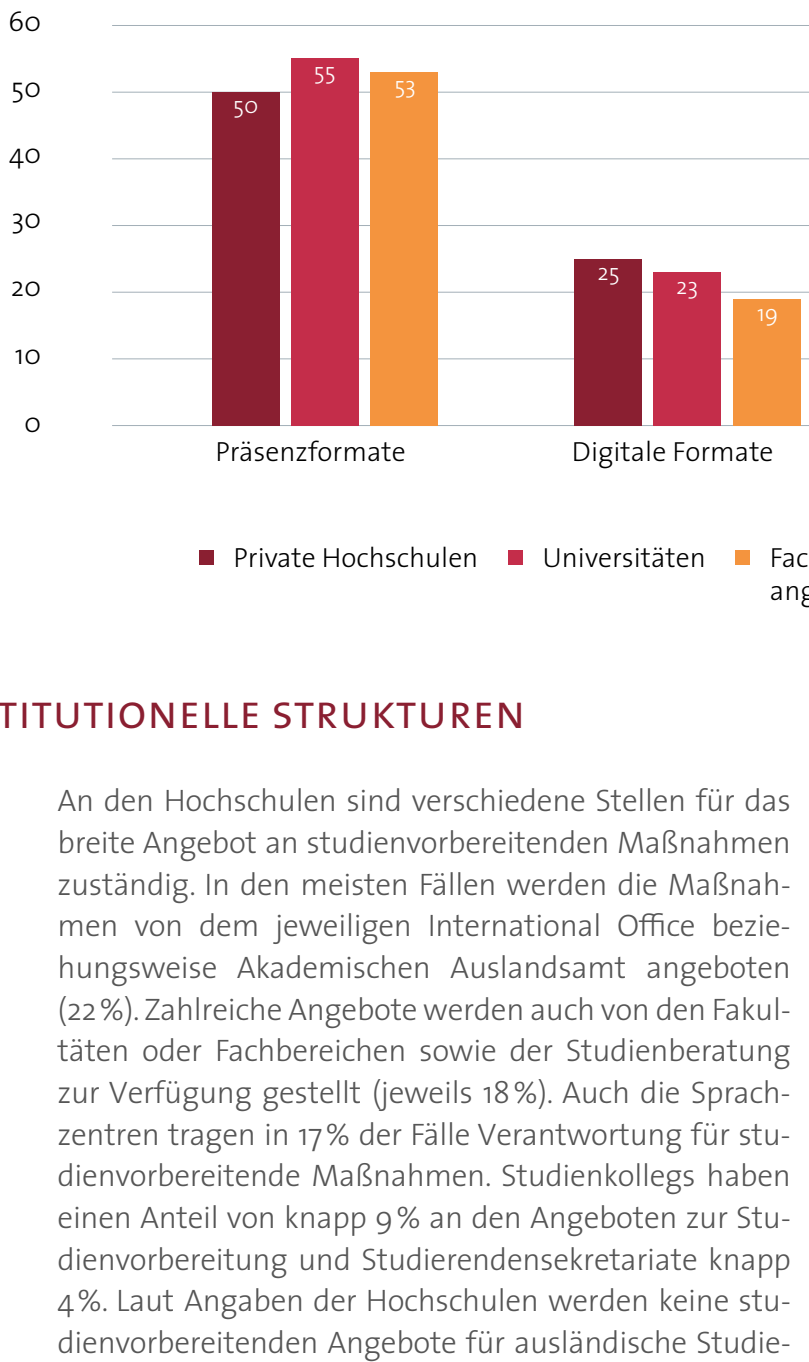

rende vom Studierendenwerk zur Verfügung gestellt. Mit circa 13\% gaben die Hochschulen unter Sonstiges an, dass zusätzliche Akteure Maßnahmen anbieten. So gibt es zum einen spezifische Projekte oder Programme, die die Studierenden auf den Studienstart vorbereiten (6) oder Lehr- bzw. Lernzentren (2), von denen einige fachlich spezialisiert sind (4). Ebenso gibt es interkulturelle (Kompetenz-)Zentren (3) sowie Bereiche der Hochschulen, die sich mit Weiterbildung beschäftigen (3). An einigen Hochschulen sind auch andere Stellen in den Prozess involviert. Hierzu zählen Stabsstellen (2), der Vizepräsident für Internationales (1), die Studiengangskoordination (1) oder der Career Service (1).

26 Allerdings gibt es in manchen Fällen einige administrative Kosten bzw. Verwaltungsgebühren, die bezahlt werden müssen. Kostenlos im Kontext dieser Publikation bedeutet die nicht erwerbswirtschaftliche Natur der Angebote. 


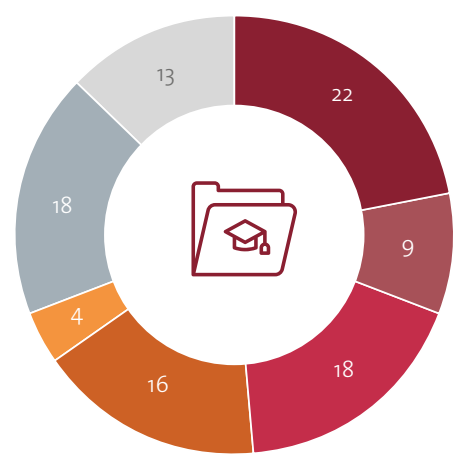

- International Office/ Akademisches Auslandsamt

- Studienkolleg

- Studierendenberatung

- Sprachzentrum

- Studierendensekretariat

- Fakultät bzw. Fachbereich

- Sonstiges

\section{ZIELGRUPPEN}

Innerhalb der Gruppe der internationalen Studierenden wurden verschiedene Zielgruppen ermittelt. Die Umfrage zeigt, dass die zahlenmäßig stärkste Zielgruppe für studienvorbereitende Maßnahmen die der geflüchteten Studierenden (27) ist. Für diese Gruppe stehen an einigen Hochschulen speziell zugeschnittene Programme und Ansprechpartnerinnen bzw. -partner (9) sowie spezielle Kurse zur sprachlichen Vorbereitung (3) zur Verfügung. Weitere relevante Zielgruppen sind Studierende mit Abschlussabsicht (24) und Austauschstudierende (18). Die Maßnahmen für Austauschstudierende konzentrieren sich stark auf Deutschsprachkurse (6) sowie interkulturelle Trainings (3). Bei Studierenden mit Abschlussabsicht werden neben Maßnahmen zur Orientierung (3) vor allem sprachbezogene Maßnahmen wie Sprachkurse (2) bzw. DSH-Kurse (2) von den Hochschulen ermöglicht. Auch das Studienkolleg (3) wurde angegeben, ebenso wie Maßnahmen zum Online-Self-Assessment (2). Grundständige Studierende (22) wurden von den Befragten häufiger genannt als Masterstudierende (15). Bei den grundständig Studierenden steht für die Hochschulen vor allem die fachliche Vorbereitung (5) im Vordergrund. Daneben spielt die sprachliche Vorbereitung eine Rolle. Ein ähnliches Bild zeichnet sich auch bei den Masterstudierenden ab: Vor allem Kurse im Zusammenhang mit der DSH (4) und fachlichen Vorbereitungen (2) werden hier von den Hochschulen genannt. Des Weiteren sind Studieninteressierte einer bestimmten Fächergruppe (12) wichtige Adressaten studienvorbereitender Maßnahmen. Hier steht bei vielen Hochschulen vor allem der MINT-Bereich (6) im Fokus. Studierende aus Nicht-EU-Staaten (10) wurden als relevantere Zielgruppe für studienvorbereitende Maßnahmen angegeben als Studierende aus EU-Staaten (4). Unter Sonstiges (8) gaben die Hochschulen unter anderem Maßnahmen an, welche Studierende aus allen Regionen gemeinsam auf das Studium vorbereiten (3).

\section{Abbildung 8}

Zielgruppen der studienvorbereitenden Maßnahmen, Angaben in absoluten Zahlen

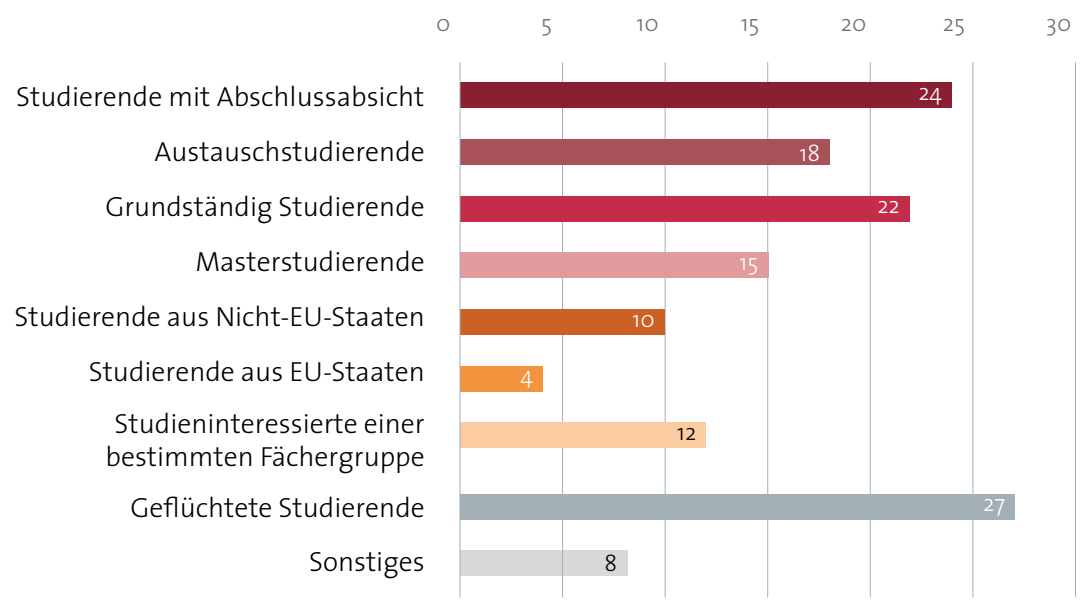




\section{HERAUSFORDERUNGEN UND BEDARFE}

Die teilnehmenden Hochschulen konnten im Fragebogen in einem Freitextfeld angeben, ob es aus ihrer Sicht Herausforderungen, Hindernisse und Bedarfe im Bereich studienvorbereitende Maßnahmen gibt. Das Freitextfeld wurde intensiv genutzt. Die Antworten wurden nach Themenbereichen geclustert, um sie auswerten zu können. Folgende Cluster wurden identifiziert: Finanzierung und Nachhaltigkeit der studienvorbereitenden Maßnahmen, rechtliche Einschränkungen/ Herausforderungen, Komplexität der institutionellen Rahmenbedingungen, sprachliche Vorbereitung, Selbsteinschätzung der Studierenden und Komplexität des Hochschulzugangs sowie digitale Elemente in der Vorbereitung.

Finanzierung und Nachhaltigkeit der studienvorbereitenden Maßnahmen

Am häufigsten wurde das Thema „Finanzierung" in Verbindung mit der Nachhaltigkeit von studienvorbereitenden Maßnahmen angesprochen. Gerade wenn solche Maßnahmen kostenlos zur Verfügung gestellt werden sollen, weisen die Hochschulen auf den Mangel an finanziellen Mitteln, vor allem für Personal, hin. Besonders die Erarbeitung digitaler oder multimedialer Inhalte zur Vorbereitung a uf ein Studium könne aus diesem Grund teilweise nicht geleistet werden. Insgesamt sei somit eine intensive Betreuung internationaler Studienanfängerinnen und -anfänger schwer zu gewährleisten. Dies wird beispielsweise bei der sprachlichen Vorbereitung deutlich, deren Finanzierung als fakultätsübergreifende Vorbereitung schwierig zu gestalten ist. Aufseiten der Studienanfängerinnen und -anfänger fehlen nach Ansicht der Befragten häufig die finanziellen Mittel, um anfallende Kursgebühren zu tragen, aber auch, um ihren Lebensunterhalt für die Dauer der studienvorbereitenden Maßnahmen zu bestreiten. Ebenfalls merken einige Hochschulen an, dass sie sich eine dauerhafte Verankerung von Fördermaßnahmen wünschen würden. Dies würde ermöglichen, einige der studienvorbereitenden Maßnahmen, die für Studierende mit Fluchthintergrund entwickelt wurden, langfristig auch für weitere Gruppen internationaler Studierender nutzbar zu machen.

\section{Rechtliche Einschränkungen/ Herausforderungen}

Als weiteres Thema wurde die Visa-Erteilung für internationale Studierende genannt. Durch langwierige Visa-Verfahren kommen internationale Studienanfän- gerinnen und -anfänger häufig nach Studienbeginn in Deutschland an. Dies wird - nach Angaben der Hochschulen - meist durch lange Wartezeiten für Termine bei den Botschaften verursacht. Dadurch ist es für die Studienanfängerinnen und -anfänger schwer, studienvorbereitende Maßnahmen vor Semesterbeginn zu absolvieren. Teilweise versuchen Hochschulen dies durch digitale Angebote, die noch im Heimatland wahrgenommen werden können, oder durch semesterbegleitende Programme auszugleichen.

Die bürokratischen Hürden wurden als ein weiteres Thema genannt, welches sich negativ auf die Studienvorbereitung auswirkt. Neben den bereits erwähnten Visaverfahren wurde kritisiert, dass häufig Vorgaben vom Jobcenter nur schwer mit der Realität der internationalen Studierenden vereinbar sind oder dass Anforderungen wie die Übersetzung von Zeugnissen nicht finanziell unterstützt werden.

\section{Komplexität der institutionellen Rahmenbedingungen}

Die Vorbereitung a uf ein Studium in Deutschland durch ein Studienkolleg wurde von den Hochschulen teilweise kritisch betrachtet. Eine zu geringe Anzahl an Studienkollegs-Plätzen steht im Verhältnis zu der Zahl der internationalen Studieninteressierten zur Verfügung. Zahlen hierzu sind schwierig zu erhalten, da die Daten in der Regel nirgends zentral erfasst werden und die staatlichen Studienkollegs den Ländern unterstehen. Auf Anfrage bei der Arbeitsgemeinschaft der Leiterinnen und Leiter der Studienkollegs an deutschen Hochschulen wurde dem Forschungsteam eine Statistik aus dem Sommersemester 2015 übermittelt. Insgesamt befanden sich 4068 internationale Studierende an Studienkollegs deutschlandweit und 2038 haben die FSP abgelegt ${ }^{27}$. Zu der Bestehensquote an Studienkollegs zeigen die Daten, die vorhanden sind, dass diese in der Regel zwischen 90 und 94\% liegt. Berichte der Arbeitsgemeinschaft der Leiterinnen und Leiter der Studienkollegs an deutschen Hochschulen zufolge hat sich die Anzahl der an Studienkolleg eingeschriebenen Studierenden in den letzten Jahren je nach Standort um zwischen $15 \%$ und $30 \%$ erhöht. Allerdings beobachtet der DAAD eine Tendenz zur Schließung von Studienkollegs in einigen Bundesländern, welches bedeutet, dass die Plätze insgesamt rückläufig sind. Einige Hochschulen versuchen den fehlenden Kapazitäten an Studienkollegs durch die Einführung innovativer Formate, wie beispielsweise Propädeutika, entgegenzuwirken. 
Sprachliche Vorbereitung

Die sprachliche Vorbereitung auf ein Studium wurde von mehreren Hochschulen als besonders wichtig für den Studienerfolg betrachtet. Diese kann besonders dann nur schwer gewährleistet werden, wenn die betreffende Hochschule über kein eigenes Sprachenzentrum verfügt. Zudem spielt auch hier die Finanzierung wieder eine zentrale Rolle: Häufig können studienvorbereitende Deutschkurse aufgrund fehlender Ressourcen nicht oder nur kosten pflichtig angeboten werden. Es könne daher ein genereller Rückgang von Deutschkenntnissen unter internationalen Studierenden beobachtet werden. Eine Differenz zwischen dem Sprachniveau laut Sprachtests und dem tatsächlichen Deutschniveau wird beklagt. Vor allem das Fehlen von fachsprachlichen Kenntnissen sehen die Hochschulen als Problem für den Studienerfolg internationaler Studierender an.

\section{Selbsteinschätzung der Studierenden und Komplexität des Hochschulzugangs}

Einige der befragten Hochschulen sehen die Selbsteinschätzung der internationalen Studierendenanfängerinnen und -anfänger bezüglich ihrer Kompetenzen als problematisch an. Diese erkennen oft nicht ihren eigenen Bedarf an studienvorbereitenden Maßnahmen und nehmen diese deshalb nicht wahr. Vor allem im Bereich des wissenschaftlichen Arbeitens und der Studienkompetenzen realisieren die Studierenden ihren Bedarf nach unterstützenden Angeboten der Hochschule häufig erst im Studienverlauf.

Einige Hochschulen merken auch an, dass der Prozess des Hochschulzugangs in Deutschland internationalen Studieninteressierten schwierig zu vermitteln ist. Häufig schrecken längere Studienzeiten durch studienvorbereitende Maßnahmen Studieninteressierte $a b$. Diese befürchten eine längere Studiendauer und sehen ggf. Probleme bei der Finanzierung über die Regelstudienzeit hinaus. Dementsprechend begrüßen einige Hochschulen die Öffnung des Hochschulzugangs durch alternative Zugangswege. Die guten Erfahrungen mit internationalen Studierenden, die über diesen Weg ein Studium in Deutschland antreten, wiegen aus Sicht der Befragten den Nachteil auf, dass das Bild des Hochschulzuganges durch die Vielzahl an Modellen noch schwerer erklärbar wird. Zudem werden dadurch Kapazitäten geschaffen, die der Überlastung der Studienkollegs entgegenwirken können. Grundsätzlich besteht bei einigen Hochschulen der Wunsch nach Schaffung von Spielräumen in der Zulassung auf Länderebene und der Feststellung der Studieneignung durch eigene Einstufungstests.

\section{Digitale Elemente in der Vorbereitung}

Einige Hochschulen arbeiten bereits mit vorgeschalteten digitalen Elementen und prüfen die fachlichen Vorkenntnisse der Studieninteressierten als Teil der Zulassung online, während diese sich noch im Heimatland befinden.

Die Problematik der verzögerten Visa-Erteilungen führt ebenfalls zu einer verstärkten Verlagerung studienvorbereitender Inhalte in digitale Formate, die noch im Heimatland abgerufen werden können. Auf diese Weise können internationale Studierende die Wartezeit auf den Aufenthaltstitel schon zur Vorbereitung auf das Studium nutzen.

Neben rein digitalen Formaten bieten die Hochschulen auch Formate mit Blended Learning oder begleitender Tutorierung an. Die Entwicklung solcher Formate wirft automatisch wieder die Ressourcenfrage auf: Studienvorbereitende Maßnahmen - ob digital oder in Präsenz - erfordern Mittel für deren Entwicklung, Durchführung und Weiterentwicklung. Die Umfrage macht deutlich, dass viele Hochschulen über zu geringe Mittel verfügen, um solche Angebote schaffen zu können. Gleichzeitig nehmen die internationalen Studienanfängerinnen und -anfänger kostenpflichtige Angebote häufig nicht wahr. Dementsprechend sind sie nicht gut genug vorbereitet, um die Anforderungen des Studiums zu bewältigen. Dies verursacht einen hohen studienbegleitenden Beratungs- und Betreuungsaufwand, der die Hochschulstrukturen stark belastet. 


\section{Praxisbeispiele an deutschen Hochschulen}

5.1 HOCHSCHULCLUSTER GROSSE UNIVERSITÄTEN

5.2 HOCHSCHULCLUSTER KLEINE UNIVERSITÄTEN

5.3 HOCHSCHULCLUSTER TECHNISCHE UNIVERSITÄTEN

5.4 HOCHSCHULCLUSTER GROSSE FACHHOCHSCHULEN

5.5 HOCHSCHULCLUSTER KLEINE FACHHOCHSCHULEN 
Im Rahmen der vorliegenden Studie wurden die teilnehmenden Hochschulen aufgefordert, eigene Praxisbeispiele studienvorbereitender Maßnahmen für internationale Studierende zu nennen. Auf einige dieser Praxisbeispiele soll im Folgenden eingegangen werden, um zu zeigen, wie die Studienvorbereitung dieser Gruppe gestaltet werden kann. Die Auswahl der Praxisbeispiele erfolgte in Anlehnung an das Kategoriensystem, welches für den Fragebogen verwendet wurde. Die von den Hochschulen angeführten Beispiele wurden hinsichtlich folgender Kategorien geclustert: sprachlich, fachsprachlich oder kulturell, obligatorisch oder freiwillig sowie kostenlos oder kostenpflichtig. Gleichzeitig wurde darauf geachtet, eine gleichmäßige Verteilung der dargestellten Praxisbeispiele an unterschiedlichen Hochschultypen zu gewährleisten. Entsprechend wur- den die Beispiele in folgende Hochschulcluster ${ }^{28}$ unterteilt: Große Universitäten ${ }^{29}$, Kleine Universitäten ${ }^{30}$, Technische Universitäten ${ }^{31}$, Große Fachhochschulen bzw. Hochschulen für angewandte Wissenschaften ${ }^{32}$ und Kleine Fachhochschulen bzw. Hochschulen für angewandte Wissenschaften ${ }^{33}$. In Bezug auf private Hochschulen konnte kein Praxisbeispiel unter den Antworten der teilnehmenden Hochschulen für die Zielgruppe der internationalen Studierenden identifiziert werden.

Nachdem für alle Kategorien und Hochschularten passende Praxisbeispiele identifiziert worden waren, wurden die jeweiligen Kontaktpersonen an den Hochschulen angesprochen und um weitere Informationen gebeten, welche die Praxisbeispiele ausführlicher darstellen. ${ }^{34}$

28 In Anlehnung an DAAD (2010) werden folgende Hochschulcluster berücksichtigt: Technische Universitäten, Große Universitäten (mehr als 20.000 Studierende), Kleine Universitäten (bis 20.000 Studierende), Große Fachhochschulen (mehr als 5.000 Studierende), Kleine Fachhochschulen (bis 5.000 Studierende) und Kunst- und Musikhochschulen. Im Rahmen dieser Untersuchung wurden Kunst- und Musikhochschulen ausgeschlossen und private Hochschulen als gesonderte Kategorie mit aufgenommen. 


\subsection{HOCHSCHULCLUSTER GROSSE UNIVERSITÄTEN}

\author{
Hochschule \\ Universität zu Köln \\ Hochschulgröße \\ 48.841 Studierende (WS 2019-2020), davon internatio- \\ nale Studierende: 3.555 (WS 2019-2020)

\section{Das Programm} \\ Studienstart International

\section{Formate} \\ Präsenz

\section{Zielgruppe(n) und Dauer} \\ Internationale Studienbewerberinnen und -bewerber \\ aus Nicht-EU-Ländern. Das Programm richtet sich an \\ Studierende in grundständigen Studiengängen und \\ erstreckt sich über ein Semester.
}

\section{Ablauf}

Die Studieneingangsphase „Studienstart International" ermöglicht Studierenden aus Nicht-EU-Ländern folgende zwei Wege des Studieneinstiegs:

Der „Studienstart International plus“ richtet sich an Studierende ohne in Deutschland anerkannte Hochschulzugangsberechtigung ( $\mathrm{HZB}$ ) und findet im Semester vor Beginn des Fachstudiums statt. Damit haben internationale Studierende die Möglichkeit, direkt mit dem Studium an der Universität zu Köln zu beginnen, ohne vorherigen Besuch eines Studienkollegs oder absolvierten Studiensemestern im Heimatland.

Den "Studienstart International integrale“ durchlaufen Studierende, die bereits über eine in Deutschland anerkannte $\mathrm{HZB}$ verfügen. Die Studierenden nehmen am Studienstart International integrale während des ersten Fachsemesters teil.

\section{Kernmerkmale}

Studienstart International beinhaltet neben studienfachspezifischen Grundlagen- bzw. Fachveranstaltungen ebenso Veranstaltungen zur Verbesserung der deutschen Sprachkompetenz für den universitären Kontext sowie der landeskundlichen Kenntnisse. Zudem nehmen die Studierenden an Seminaren zur Förderung von Studienkompetenzen und interkultureller Sensibilisierung teil. Regelmäßige Informationsund Orientierungsveranstaltungen bringen ihnen die verschiedenen universitären Einrichtungen sowie andere universitätsnahe Beratungseinrichtungen nahe. Die Universität zu Köln verfolgt mit Studienstart International als wesentliche Ziele die Verbesserung der Studienbedingungen für internationale Studierende, ihre Integration in das Studienumfeld, die Senkung der Abbruchquoten sowie die Steigerung ihres Studienerfolgs.

\section{Ansprechperson}

Britta Schlüter de Castro \& Anastasija Pitko

E-Mail

studienstart-international@verw.uni-koeln.de

Projekthomepage

www.si.uni-koeln.de 


\subsection{HOCHSCHULCLUSTER KLEINE UNIVERSITÄTEN}

Hochschule

Universität des Saarlandes

\section{Hochschulgröße}

16.837 Studierende (WS 2019-2020), davon internationale Studierende: 3.128 (WS 2019-2020)

\section{Das Programm}

VSIMINT (Vorbereitungsstudium International speziell für Studienbewerber und -bewerberinnen ohne direkte Hochschulzugangsberechtigung)

Formate

Präsenz

\section{Zielgruppe(n) und Dauer}

Internationale Studienbewerberinnen und -bewerber ohne direkte Hochschulzugangsberechtigung, die natur- und ingenieurwissenschaftliche Studiengänge im Saarland studieren möchten. Das Studium beginnt mit einer zweisemestrigen Orientierungsphase, in der die Teilnehmenden flexibel zwischen acht Fächern (Physik, Chemie, Mathematik, Informatik, Computerlinguistik, Materialwissenschaften und Werkstofftechnik, Systems Engineering oder Mikrotechnologie und Nanostrukturen) auswählen und bereits Credit Points erwerben können. Vorgeschaltet sind je nach Kenntnisstand ein oder zwei vorbereitende Semester.

\section{Ablauf}

1. Semester: Sofern das entsprechende Sprachniveau (B2 nach GER) durch den Einstufungstest nachgewiesen werden konnte, lernen die Bewerberinnen und Bewerber ein Semester lang Deutsch (18 SWS), Mathematik (10 SWS) und Physik (10 SWS). Das Semester schließt mit einem Deutschtest (identisch mit der lokalen DSH-Prüfung) und der Abschlussklausur in beiden MINT-Fächern ab.

Sollten die Deutschkenntnisse noch nicht ausreichend sein, können die Teilnehmenden zunächst die kostenfreien Deutschkurse des ISZ Saar belegen und dann später in das 1. Semester des VSiMINT aufsteigen.

2. und 3. Semester: Probestudium im Fachbereich Bachelor Plus MINT mit den Pflichtfächern „Höhere Mathematik für Ingenieure“, „Fachsprache Technik“ sowie einer Auswahl weiterer Wahlfächer und Orientierungs- angebote. Bestandene Klausuren können im späteren Bachelorstudium angerechnet werden. Ziel ist es, nach dem VSiMINT das Bachelorstudium rasch und erfolgreich zu durchlaufen.

\section{Kernmerkmale}

Die Kombination von Studienvorbereitung und Probestudium bringt Studieninteressierte schneller an die Universität, da sie sich nur einmal bewerben müssen und bei erfolgreichem Verlauf garantiert in den Studiengang Bachelor Plus MINT einsteigen können. Das Probestudium ist genau auf die Interessen und Bedürfnisse der Teilnehmenden abgestimmt und kann flexibel gestaltet werden. Diese Orientierungsphase wird im Vorbereitungsstudium mit weiteren Unterstützungsprogrammen für internationale Bewerberinnen und Bewerber angereichert, damit sie einen Überblick über die vielfältigen Studienmöglichkeiten im Saarland erhalten, bevor sie sich festlegen. Es gibt vielfältige Möglichkeiten, schon im Probestudium Credit Points für das Fachstudium zu sammeln und so Zeit zu sparen.

\section{Ansprechperson \\ Dr. Michael Aulbach \\ info@isz.uni-saarland.de}

\section{Projekthomepage}

https://www.uni-saarland.de/einrichtung/isz-saar/ vsi-mint.html 


\subsection{HOCHSCHULCLUSTER TECHNISCHE UNIVERSITÄTEN}

\section{Hochschule}

Rheinisch-Westfälische Technische Hochschule Aachen (RWTH) Aachen

\section{Hochschulgröße}

45.628 Studierende (WS 2019-2020), davon internationale Studierende: 11.280 (WS 2019-2020)

\section{Das Programm}

Fast Track Bachelor Admission - FTBA

\section{Formate}

Präsenz

\section{Zielgruppe(n) und Dauer}

Internationale Studienbewerber und -bewerberinnen ohne direkte Hochschulzugangsberechtigung. Jede neue Kohorte beginnt das Studium im Wintersemester des jeweiligen Jahres. Die Betreuungsangebote sind nur für das erste Fachsemester vorgesehen.

\section{Ablauf}

Die ausgewählten Teilnehmerinnen und Teilnehmer nehmen an einem Betreuungsprogramm teil, welches sie fachlich, sprachlich und kulturell auf das Studium vorbereitet. Das Programm beinhaltet

- die persönliche Betreuung während des gesamten Studiums

- die Unterbringung in einem Studierendenwohnheim im ersten Fachsemester

- die Vermittlung an einen erfahrenen Studierenden der RWTH

- programmspezifische Veranstaltungen

- einen studienvorbereitenden MINTFachsprachkurs

- die Teilnahme an Vorkursen im Bereich Mathematik, Chemie und Informatik

- die Betreuungsangebote der Welcome Week für internationale Studierende

- einen semesterbegleitenden Fachsprachkurs im ersten Fachsemester

- ein semesterbegleitendes Fachtutorium im ersten Fachsemester

\section{Kernmerkmale}

Um am FTBA-Programm teilnehmen zu können, müssen Studienbewerber und -bewerberinnen überdurchschnittliche Schulleistungen, hervorragende TestAS-Ergebnisse und solide Deutschkenntnisse nachweisen. Aktuell ist nur eine Bewerbung in den folgenden Fächern möglich: Maschinenbau, Wirtschaftsingenieurwesen Fachrichtung Maschinenbau sowie Computational Engineering Science.

\section{Ansprechperson}

Azadeh Hartmann-Alampour

Azadeh.Hartmann-Alampour@zhv.rwth-aachen.de

\section{Projekthomepage}

https://www.rwth-aachen.de/cms/root/Studium/ Vor-dem-Studium/Zugangsvoraussetzungen/ Besonderheiten-internationale-Studienint/ crvm/ Fast-Track-Bachelor-Admission/ 


\subsection{HOCHSCHULCLUSTER GROSSE FACHHOCHSCHULEN}

Hochschule

Hochschule für Angewandte Wissenschaften (HAW)

Hamburg

\section{Hochschulgröße}

17.049 Studierende (WS 2019-2020), davon internationale Studierende: 2.500 (WS 2019-2020)

\section{Das Programm \\ Propädeutikum}

\section{Formate}

Präsenz

\section{Zielgruppe(n) und Dauer}

Das Programm richtet sich an Studienbewerberinnen und -bewerber aus Nicht-EU-Ländern, die bereits eine Hochschulzulassung für eine der Hamburger Hochschulen haben und anschließend ein Bachelorstudium beginnen wollen. Wer in einem EU-Land die Hochschulzugangsberechtigung erworben hat und an der HAW Hamburg studieren möchte, kann sich ebenfalls für das Propädeutikum bewerben. Es findet in jedem Sommersemester statt, so dass die Teilnehmerinnen und Teilnehmer im folgenden Wintersemester mit dem regulären Studium beginnen können. Die Propädeutikkurse finden von Mitte März bis Ende Juni bzw. Mitte Juli am Studienkolleg Hamburg statt.

\section{Ablauf}

Die Teilnehmerinnen und Teilnehmer werden sprachlich (in Deutsch, z.T. auch in Englisch) gefördert und inhaltlich auf das Fachstudium vorbereitet. Ein weiterer Schwerpunkt liegt auf der Einführung in die Methodik des Studiums in Deutschland. Beispielsweise werden:

- Präsentationen unter Einsatz moderner Medien erarbeitet und gehalten

- Referate ausgearbeitet und vorgetragen

- Diskussion, Bewertung und Stellungnahme geübt

- Grafiken und Tabellen sprachlich umgesetzt und ausgewertet

- Texterschließung und Recherche geübt sowie

- in den naturwissenschaftlichen Kursen Experimente geplant, durchgeführt und ausgewertet.

\section{Kernmerkmale}

Ziel des Propädeutikums ist es, die Teilnehmerinnen und Teilnehmer fachlich, methodisch und sprachlich so auf das Studium vorzubereiten, dass der gewünschte Studiengang anschließend erfolgreich und ohne Zeitverlust absolviert werden kann. Das Propädeutikum bietet eine gezielte fachliche und sprachliche Vorbereitung auf das Studium, eine Einführung in die Studienorganisation und eine Einführung in die interkulturelle Kommunikation auf dem Campus. Es wird für die folgenden Fächergruppenangeboten: Wirtschaftswissenschaften und Soziale Arbeit, Ingenieurwissenschaften, Geistes- und Kulturwissenschaften, Mathematik, Informatik und Naturwissenschaften.

\section{Ansprechperson \\ Dr. Nancy Falke \\ propaedeutikum@haw-hamburg.de}

\section{Projekthomepage}

http://www.studienkolleg-hamburg.de/

propaedeutik/ 


\subsection{HOCHSCHULCLUSTER KLEINE FACHHOCHSCHULEN}

\author{
Hochschule \\ Alice Salomon Hochschule Berlin (ASH) \\ Hochschulgröße \\ 4.047 Studierende (WS 2019-2020), davon internatio- \\ nale Studierende: 418 (WS 2019-2O2O) \\ Das Programm \\ ASH Pre-Study-Programm \\ Formate \\ Präsenz

\section{Zielgruppe(n) und Dauer} \\ Das ASH Pre-Study-Programm richtet sich an studien- \\ interessierte Menschen mit Fluchterfahrung, die eine \\ direkte Hochschulzugangsberechtigung haben, sich \\ aber noch nicht erfolgreich für die Studiengänge an \\ der ASH Berlin bewerben können. Im Rahmen des ein- \\ jährigen Programms absolvieren die Teilnehmerinnen \\ und Teilnehmer den Deutschsprachkurs und ein drei- \\ monatiges Vorpraktikum im gewünschten Berufsfeld.
}

\section{Ablauf}

Das ASH Pre-Study Programm dauert ein Jahr (von Oktober bis September) und besteht aus:

- Programm

- Deutsch- und Studienvorbereitungskurs (Niveau B1 bis $C_{1}$ )

- Belegung erster Seminare

- Absolvierung weiterer studienspezifischer Zugangsvoraussetzungen (u.a. Ableistung eines Vorpraktikums)

- Begleitung

- Unterstützung und Begleitung durch Mentorinnen und Mentoren

- Abschließende Unterstützung bei der Bewerbung ins reguläre Studium

\section{Kernmerkmale}

Das ASH Pre-Study Programm richtet sich an Menschen mit Fluchterfahrung, die sich noch nicht für ein reguläres Studium an der ASH Berlin bewerben können. Unter anderem können im Rahmen des zweisemestrigen Pre-Study Programms sprachliche Kompetenzen in Deutsch und das für manche Studiengänge verpflichtende Vorpraktikum erworben werden. Zusätzlich besteht die Möglichkeit, erste Kurse zu belegen, die im Studium angerechnet werden. Das Programm ist kostenlos, Fahrtkosten werden selbst getragen.

\section{Ansprechperson}

Silvia Ben Mahrez

benmahrez@ash-berlin.eu

\section{Projekthomepage}

https://www.ash-berlin.eu/studium/studiengaenge/ ash-pre-studies-for-refugees/ 
Fazit und Ausblick 
Die vorliegende Studie unternimmt eine explorative Analyse von aktuellen Maßnahmen zur Studienvorbereitung, die für internationale Studierende an deutschen Hochschulen angeboten werden. Zwischen Mai 2019 und Januar 2020 wurde eine Befragung von Mitarbeiterinnen und Mitarbeitern deutscher Hochschulen konzipiert und durchgeführt. Ziel war es, Antworten auf die Frage zu finden, welche studienvorbereitenden Maßnahmen deutsche Hochschulen ergreifen, um ihre internationalen Studierenden bei der Bewältigung ihrer Anpassungsschwierigkeiten in Deutschland zu unterstützen.
Die Ergebnisse der Untersuchung geben einen Überblick über Angebote, Formate, institutionelle Strukturen, Zielgruppen sowie Herausforderungen und Bedarfe. Angesichts der Tatsache, dass diese Publikation nur einen ersten Überblick bietet, bleiben eine Reihe von Fragen offen. Hierbei geht es etwa um die Messbarkeit der Effektivität der Maßnahmen, insbesondere mit Blick auf die Erfahrung, die Zufriedenheit, sowie den Studienerfolg der Studierenden. Eine Folgeuntersuchung könnte eine differenzierte Betrachtung der Maßnahmen in Bezug auf Voraussetzungen, Dauer, Monitoringsysteme, Kosten, personelle Ressourcen und inhaltliche Unterschiede ermöglichen.

\section{AUSBLICK}

Im Rahmen dieser Publikation wurden ausschließlich Maßnahmen analysiert und dargestellt, die für internationale Studierende in Deutschland angeboten werden. Ähnlichkeiten lassen sich unabhängig von Hochschulgröße und -profil beobachten. Es lässt sich jedoch feststellen, dass die Zahl der alternativen Vorbereitungsangebote im Heimatland der Studierenden zunimmt, begründet zum Beispiel in langen Visa-Bearbeitungszeiten. Zu beobachten ist zudem ein Trend zu im Ausland von deutschen Hochschulen angebotenen Studienkollegs. Ein erfolgreiches Beispiel für die Studienvorbereitung im Heimatland stellt das Programm „Studienbrücke“ dar, welches seit 2015 angeboten wird. Ziel der „Studienbrücke“ ist es, zu einem sehr frühen Zeitpunkt - teilweise bereits in der 9. Klasse - leistungsfähige Schülerinnen und Schüler aus unterschiedlichen Regionen der Welt anzusprechen und sie systematisch auf ein Studium in den MINT-Fächern in Deutschland vorzubereiten.
Viele Initiativen werden nach wie vor analog durchgeführt und somit die Chancen der Digitalisierung zur zeitsparenden und kostengünstigen Studienvorbereitung vor der Ankunft in Deutschland noch nicht genutzt. Diese Studie hat gezeigt, dass insgesamt 21\% der Angebote der teilnehmenden Hochschulen digital konzipiert sind. Es kann davon ausgegangen werden, dass der Anteil der digitalen Angebote sowie Blended Learning-Angebote in den kommenden Jahren zunehmen wird. Es ist zu erwarten, dass hierbei die Personalisierung der Betreuung und Begleitung von Studierenden eine prominentere Rolle spielen wird ${ }^{35}$. Durch digitale Maßnahmen können Studierende in allen Phasen ihres Bildungsprozesses wie Entdeckung, Bewerbung, Entscheidung, Vorbereitung, Ankommen, Studieneinstieg bis hin zum Abschluss wirksam unterstützt werden. Dies könnte es Hochschulen und Hochschulsystemen ermöglichen, nicht nur auf eine heterogene Bewerberlage und die entsprechenden Herausforderungen zu reagieren, sondern auch eine neue Generation von Studierenden mit neuen Bedürfnissen und Erwartungen zu erreichen und zu begleiten.

35 Ein Beispiel dieser Entwicklung stellt das BMBF-geförderte Projekt „Digitaler Campus“ dar, welches seit Februar 2020 läuft. Das Projekt hat es sich zum Ziel gesetzt, ein Portal vernetzter Plattformservices zur Information, Anwerbung, Rekrutierung sowie sprachlichen, fachlichen und kulturellen Vorbereitung internationaler Studierender zu entwickeln. (siehe www.daad.de/digitalercampus) 
Methodische Durchführung der Studie 


\section{Rekrutierung und Stichprobe}

Als Grundlage für die zu befragenden Hochschulen wurde der Verteiler der Mitgliedshochschulen der Hochschulrektorenkonferenz genutzt. Dieser umfasst 288 Hochschulen. Bei der Selektion der zu befragenden Hochschulen wurde entschieden, folgende Hochschulen aus der Stichprobe zu entfernen:

- Kunst- und Musikhochschulen

- Theologische/ Katholische Hochschulen

- Spezialisierte Hochschulen wie beispielsweise Hochschulen für Forstwirtschaft

Nach der Bereinigung wurden 210 Hochschulen für die Untersuchung berücksichtigt.

\section{Technische Umsetzung der Online-Umfrage ${ }^{36}$}

Der Fragebogen wurde in dem Umfragetool Limesurvey programmiert. Durch bedingte Antworten variierte der Fragebogen für die Hochschulen ja nach Antwortverhalten. Es wurde mit binären Antworten, Kategoriensystemen, Multiple Choice und verschiedenen Formen von Freitexten gearbeitet, um sowohl quantitative als auch qualitative Antworten der Hochschulen zu ermöglichen.

\section{Feldphasenmanagement und Versandaktivitäten}

Der Fragebogen wurde Anfang Oktober 2019 an die aus dem Verteiler der Hochschulrektorenkonferenz gewonnenen Kontaktdaten verschickt. Die Hochschulen erhielten eine Einladungsmail, die den Hintergrund und das Ziel der Studie erläuterte und einen Link zum Onlinefragebogen enthielt. Als Frist für die Beantwortung der Umfrage wurde das Monatsende genannt. Einige Hochschulen meldeten auf die Einladung zur Umfrage die passenden Ansprechpartnerinnen und - partner zurück, diese wurden daraufhin nachträglich zur Studie eingeladen. In der Mail wurde ein Funktionspostfach des DAAD angegeben (svm@daad.de ${ }^{37}$ ), an welches sich die Hochschulen bei Rückfragen wenden konnten. Einige Hochschulen wollten den Fragebogen vorab sehen, um beurteilen zu können, welche Stellen für die Beantwortung zuständig sein könnten. In diesen Fällen wurde der Fragebogen als Ganzes in Form eines PDF-Dokuments zur Verfügung gestellt. In einzelnen Fällen beantworteten mehrere Personen einer Hochschule den Fragebogen und diese Antworten wurden zusammengeführt. Eine Woche vor Fristende wurde an die Hochschulen, die noch nicht geantwortet hatten, eine Erinnerung zur Teilnahme an der Umfrage geschickt. Die ursprüngliche Frist wurde für einige Hochschulen auf Wunsch verlängert. Als letzte Frist wurde das Ende der ersten Novemberwoche festgesetzt. 


\section{LITERATURVERZEICHNIS}

Asdonk, J.; Kuhnen, S.; Bornkessel, P. (Hrsg.). (2013). Von der Schule zur Hochschule. Analysen, Konzeptionen und Gestaltungsperspektiven des Übergangs. Waxmann; Auflage: 1 (29. April 2013).

Banscherus, U.; Wolter, A.; Kamm, C.; Otto, A.; Spexard, A. (2014). Durchlässigkeit zwischen beruflicher und akademischer Bildung als mehrstufiges Konzept: Bilanz und Perspektiven. Beiträge zur Hochschulforschung. 34. 72-91.

Bechtold, G. \& Helferich, P. (Hrsg.) (2008). Generation Bologna: Neue Herausforderungen am Übergang Schule-Hochschule. wbv Media; Auflage: 1 (10. Juni 2008).

Bellenberg, G. \& Forell, M. (Hrsg.) (2013). Bildungsübergänge gestalten: Ein Dialog zwischen Wissenschaft und Praxis. Waxmann Verlag GmbH, Münster 2013.

Borgwardt, A.; John-Ohnesorg, M. \& Zöllner, J. (2015). Hochschulzugang für Flüchtlinge - aktuelle Regelungen in den Bundesländern. Ergebnisse einer Umfrage unter den für Hochschulen zuständigen Landesministerien, Stand 25. August 2015. Berlin: Friedrich-EbertStiftung, Abt. Studienförderung (Hochschulpolitik).

Bornkessel, P. \& Jupp, A. (Hrsg.) (2011). Der Übergang Schule- Hochschule: Zur Bedeutung sozialer, persönlicher und institutioneller Faktoren am Ende der Sekundarstufe II. VS Verlag für Sozialwissenschaften.

British Council. (2019). The shape of global higher education: International comparisons with Europe. International Higher Education.

Brügelmann, H. \& Heymann, H. (2002) PISA 2000: Befunde, Folgerungen. Zum internationalen Bericht der OECD. In: Pädagogik 54. Jg., 2002, Heft 3. S.41-43.

DAAD \& DZHW (2019). Wissenschaft weltoffen kompakt: Daten und Fakten zur Internationalität von Studium und Forschung in Deutschland. mbv Media.
Dahm, G. \& Kerst, C. (2013). Immer noch eine Ausnahme - nicht-traditionelle Studierende an deutschen Hochschulen. Zeitschrift für Beratung und Studium, 2, 2013, 34-39.

Erdmenger, T. \& Pasternack, P. (2013). Eingänge und Ausgänge: Die Schnittstellen der Hochschulbildung in Sachsen-Anhalt (HoF-Arbeitsbericht 2013). Institut für Hochschulforschung (HoF) an der Martin-Luther-Universität, Halle-Wittenberg, 2013.

Falk, S., Thies, T., Yildirim, H., Zimmermann, J., Kercher, J. \& Pineda, J. (2019): Methodenbericht zur Studie „Studienerfolg und Studienabbruch bei Bildungsausländern in Deutschland im Bachelor- und Masterstudium" (Sesaba). Dokumentation der Welle o bis 2. Release 1.

Gaus, D. (2019). Gestaltung von Übergängen ins Studium als Bestandteil eines hochschulischen Bildungsauftrages? Versuch einer historisch-systematischen Themeneinordnung. In: Driesen, C. \& Ittel, A.(Hrsg.) (2019). Der Übergang in die Hochschule: Strategien, Organisationsstrukturen und Best Practices an deutschen Hochschulen. Waxmann; Auflage: 1 (7. Januar 2019).

Gemeinsame Wissenschaftskonferenz (Hrsg.) (2013). Strategie der Wissenschaftsminister/innen von Bund und Ländern für die Internationalisierung der Hochschulen in Deutschland (Beschluss der 18. Sitzung der Gemeinsamen Wissenschaftskonferenz am 12. April 2013 in Berlin). Berlin. Verfügbar unter: https://www.bmbf.de/files/ aaalnternationalisierungsstrategie_GWKBeschluss_12_04_13.pdf. (Abgerufen am 21.01.2020)

Grüttner, M., Schröder, S., Berg, J., Otto, C. (2018). Die Situation von Geflüchteten auf dem Weg ins Studium. (DZHW Brief 5|2018). Hannover: DZHW.

Hansel, T. (Hrsg.) (2003). Pisa - und die Folgen? Die Wirkung von Leistungsvergleichsstudien in der Schule. Centaurus Verlag \& Media; Auflage: 1. Aufl. 2003 (31. Dezember 2003).

DAAD (2010) „Internationalität an deutschen Hochschulen - Konzeption und Erhebung von Profildaten". Band 65. Dok \& MAT 
Hell, B. (2007): Die Prozentrangnormierung als Alternative zum bisherigen Umrechnungsverfahren ausländischer Sekundarabschlussnoten für die Verwendung in den Zulassungsverfahren deutscher Hochschulen. Hochschulmanagement, 4, S. 93-99.

Heublein, U. \& Schmelzer, R. (2018): Die Entwicklung der Studienabbruchquoten an den deutschen Hochschulen: Berechnungen auf Basis des Absolventenjahrgangs 2016. Hannover: DZHW.

Hill, L. und Key, O. (2019). Orientierung und Unterstützung zum Studieneingang: Umsetzungsstand an deutschen Hochschulen. Arbeitspapier Nr. 226 des CHE Centrum für Hochschulentwicklung, Gütersloh, September 2019.

Hoffmeyer-Zlotnik, P. \& Grote, J. (2019). Anwerbung und Bindung von internationalen Studierenden in Deutschland. Studie der deutschen nationalen Kontaktstelle für das Europäische Migrationsnetzwerk (EMN). Working Paper 85. Bundesamt für Migration und Flüchtlinge 2019.

Langer, R. (2008a): Warum haben die PISA gemacht? Ein Bericht über einen emergenten Effekt internationaler politischer Auseinandersetzungen. In: Langer, R. (Hrsg.): Warum tun die das? Governanceanalysen zum Steuerungshandeln in der Schulentwicklung Wiesbaden. VS Verlag für Sozialwissenschaften. S. 49-72.

Lin-Klitzing, D.; Di Fuccia, D. \& Stengl-Jörns, R. (Hrsg.) (2014). Abitur und Studierfähigkeit: Ein interdisziplinärer Dialog (S. 55-73). (Gymnasium - Bildung - Gesellschaft). Bad Heilbrunn: Klinkhardt.

Lokhande, M. (2017): Vom Hörsaal in den Betrieb? Internationale Studierende beim Berufseinstieg in Deutschland. SVR-Forschungsbereich, Berlin.

Loy, C.; Hell, B. \& Robra, B. (2009). Priorisierung ausländischer Bildungsabschlüsse bei der Zulassung zum Medizinstudium. Hochschulwesen; 57 (2009), S. 98-102.

Morris-Lange, S. (2019): Dem demografischen Wandel entgegen. Wie schrumpfende Hochschulstandorte internationale Studierende gewinnen und halten. SVR-Forschungsbereich, Berlin.
Morris-Lange, S. (2017): Allein durch den Hochschuldschungel. Hürden zum Studienerfolg für internationale Studierende und Studierende mit Migrationshintergrund. SVR-Forschungsbereich, Berlin.

Morris-Lange, S. \& Brands, F. (2015): Zugangstor Hochschule: Internationale Studierende als Fachkräfte von morgen gewinnen. SVR-Forschungsbereich, Berlin.

Morris-Lange, S.; Brands, F. \& Crysmann, T. (2015): Deutschland als attraktiver Arbeitsmarkt für internationale Studierende? Bleibeabsichten, Verbleib und Berufseinstiegshürden einer begehrten Zuwanderergruppe. In Barz, H. \& Jung, M. Ausländische Fachkräfte gesucht - Voreilig? Notwendig? Willkommen? Düsseldorf University Press, Düsseldorf.

OECD (2009). Higher Education to 2030, Volumen 2: Globalisation OECD Publishing, Paris.

Pasternack, P. (2019). Von der Schule ins Studium: Analytische und empirische Unterscheidungen. In: Driesen, C. \& Ittel, A. (Hrsg.) (2019). Der Übergang in die Hochschule: Strategien, Organisationsstrukturen und Best Practices an deutschen Hochschulen. Waxmann; Auflage: 1 (7. Januar 2019).

Schellhorn, S.; Frank, M. \& Kreitz, C. (2019). Brückenkurse für mathematische und informatiknahe Studiengänge. In: Schubarth, W.; Mauermeister, S.; SchulzeReichelt, F. \& Seidel, A. (Hrsg.) (2019). Alles auf Anfang!: Befunde und Perspektiven zum Studieneingang. Universitätsverlag Potsdam, 2019. Potsdamer Beiträge zur Hochschulforschung, 4 .

Trautwein, U. (2013). Übergang zwischen Schule und Hochschule - empirische Befundlage. In: Bellenberg, G. \& Forell, M. (Hrsg.) (2013). Bildungsübergänge gestalten: Ein Dialog zwischen Wissenschaft und Praxis. Waxmann Verlag GmbH, Münster 2013.

Wisniewski, K. (2018). Sprache und Studienerfolg von Bildungsausländerinnen und -ausländern: Eine Längsschnittstudie an den Universitäten Leipzig und Würzburg. Informationen Deutsch Als Fremdsprache, 45(4), 573-597. 
Anhang

1

Anhang

पn<smiles>C1CCC1</smiles>

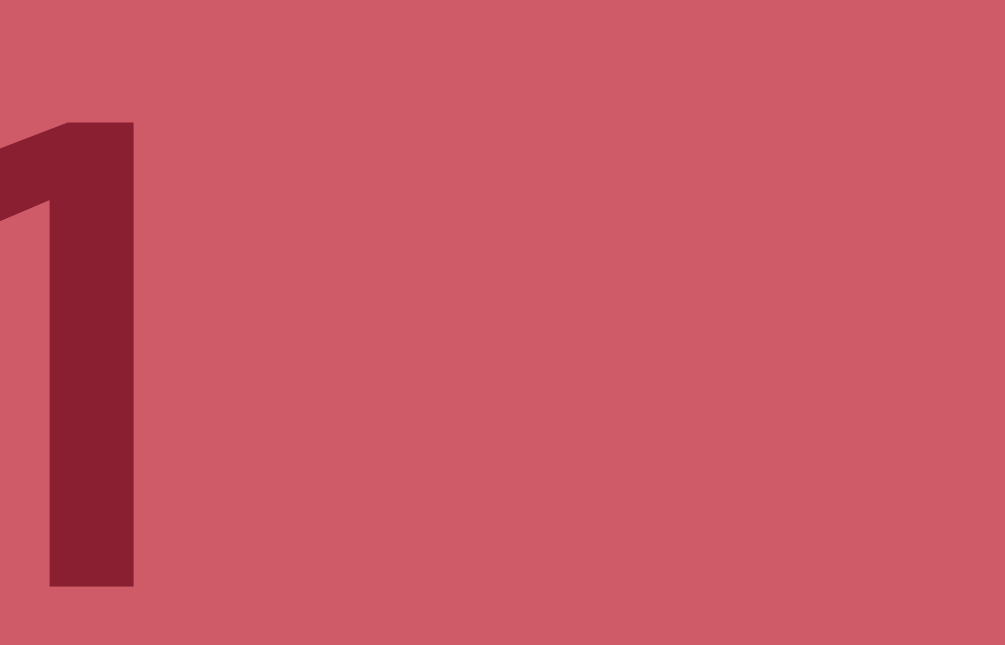

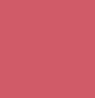




\section{STUDIENKOLLEGS IN DEUTSCHLAND}

\begin{tabular}{|c|c|c|c|c|}
\hline Bundesland & Stadt & Name des Studienkollegs & privat/staatlich & Uni/FH \\
\hline \multirow[t]{3}{*}{ Baden-Württemberg } & Heidelberg & Studienkolleg an der Universität Heidelberg & staatlich & Uni \\
\hline & Karlsruhe & $\begin{array}{l}\text { Studienkolleg des KIT (Karlsruher Institut } \\
\text { für Technologie) }\end{array}$ & staatlich & Uni \\
\hline & Konstanz & Studienkolleg an der HTWG Konstanz & staatlich & $\mathrm{FH}$ \\
\hline \multirow[t]{2}{*}{ Bayern } & München & $\begin{array}{l}\text { Studienkolleg bei den Universitäten des } \\
\text { Freistaates Bayern (in München) }\end{array}$ & staatlich & Uni \\
\hline & Coburg & $\begin{array}{l}\text { Studienkolleg bei den Fachhochschulen des } \\
\text { Freistaates Bayern (in Coburg) }\end{array}$ & staatlich & $\mathrm{FH}$ \\
\hline \multirow[t]{2}{*}{ Berlin } & Berlin & Studienkolleg an der FU Berlin & staatlich & Uni \\
\hline & Berlin & Studienkolleg an der FU Berlin & staatlich & Uni \\
\hline Hamburg & Hamburg & Studienkolleg an der Universität Hamburg & staatlich & Uni \\
\hline Hessen & Darmstadt & Studienkolleg an der TU-Darmstadt & staatlich & Uni ur \\
\hline
\end{tabular}

\begin{tabular}{llll}
\hline Frankfurt am & Studienkolleg an der Goethe-Universität & staatlich & Uni und FH \\
Main &
\end{tabular}

Kassel Studienkolleg an der Universität Kassel $\quad$ staatlich $\quad$ Uni und FH

\begin{tabular}{lll}
\hline Marburg & Studienkolleg Mittelhessen der & staatlich
\end{tabular}

\begin{tabular}{lllll}
\hline $\begin{array}{l}\text { Mecklenburg- } \\
\text { Vorpommern }\end{array}$ & Wismar & $\begin{array}{l}\text { Studienkolleg an der Hochschule } \\
\text { Wismar }\end{array}$ & staatlich & FH \\
\hline Niedersachsen & Hannover & $\begin{array}{l}\text { Studienkolleg an der Universität } \\
\text { Hannover }\end{array}$ & staatlich Uni & \\
\hline Nordrhein-Westfalen & Bochum & $\begin{array}{l}\text { Studienkolleg des Ökumenischen } \\
\text { Studienwerks e.V. }\end{array}$ & $\begin{array}{l}\text { privat (staatlich } \\
\text { genehmigt) }\end{array}$
\end{tabular}


Bewerbung auf Studiengang an Hochschule in BaWü. Hochschule entscheidet über Zuteilung zum Studienkolleg Heidelberg.
Verwaltungsbeitrag von $150 € \quad 2$ pro Semester
$\mathrm{B}$

\section{B1}

pro Semester
B1 pro Semester

Verwaltungsgebühr von $50 € \quad 2$ pro Semester und Immatrikulations-gebühr von ca. $120 €$ je nach Studiengang und Hochschule Prüfung der Unterlagen durch HS und Zuteilung zum Studienkolleg München

$105 €$ pro Semester

2 $\mathrm{B} 1+/ \mathrm{B} 2$ Zeugnisanerkennung durch die gewünschte Hoch
danach Bewerbung beim Studienkolleg Coburg Bewerbung direkt am Studienkolleg oder über eine $305 €$ Semestergebühren 2 Hochschule

$\begin{array}{lll}\text { Semesterbeitrag von ca. } 310 € & 2 & \text { B2 } \\ \text { Semesterbeitrag von ca. } 330 € & 2 & \text { B2 }\end{array}$

Studienkolleg

Bewerbung direkt an den Hochschulen TU Darmstadt, keine Kosten, aber $100 €$ 2 Hochschule Darmstadt, RheinMain University of Applied Gebühr für FSP Sciences, Hochschule Geisenheim oder Evangelische Hochschule Darmstadt (EHD)

Bewerbung bei der Goethe-Universität, der Frankfurt Semesterbeitrag von ca. $370 € \quad 2$ University of Applied Sciences oder Hochschule für Gestaltung in Offenbach, der Hochschule St. Georgen oder der Lutherischen Theologischen Hochschule Oberursel.

Bewerbung nur für Studiengänge der Universität Kassel Semesterbeitrag von ca. $290 € \quad 2$ und der FH Fulda über uni-assist. Uni-assist übernimmt Zuteilung zum Studienkolleg

Bewerbung für einen Studiengang an den Hochschulen TH Mittelhessen, Universität Gießen, Universität Marburg über uni-assist. Anschließend erfolgt die Zuteilung zum Studienkolleg.

Bewerbung direkt am Studienkolleg der Hochschule. Zulassung nach Aufnahmetest

Bewerbung direkt an niedersächsischer Hochschule. Zuteilung zum Studienkolleg erfolgt durch HS Bewerbung direkt am Studienkolleg
Semesterbeitrag von ca.110€ i.d.R. 2

B2

Studiengebühren: $250 €+\quad 2$ Semesterbeitrag: $300 €$

Studierende des Studienkol2 legs werden an der Universität Bochum eingeschrieben und zahlen einen Sozialbeitrag von ca. $300 €$ pro Semester 


\begin{tabular}{|c|c|c|c|c|}
\hline Bundesland & Stadt & Name des Studienkollegs & privat/staatlich & Uni/FH \\
\hline \multirow[t]{2}{*}{ Nordrhein-Westfalen } & Mettingen & $\begin{array}{l}\text { Studienkolleg Mettingen } \\
\text { (Luso-brasilianisches Studienkolleg) }\end{array}$ & $\begin{array}{l}\text { privat (staatlich } \\
\text { anerkannt) }\end{array}$ & \\
\hline & Paderborn & $\begin{array}{l}\text { Internationales Studienkolleg an der } \\
\text { Universität Paderborn }\end{array}$ & $\begin{array}{l}\text { privat (staatlich } \\
\text { anerkannt) }\end{array}$ & \\
\hline \multirow[t]{2}{*}{ Rheinland-Pfalz } & Mainz & $\begin{array}{l}\text { Studienkolleg der Johannes Gutenberg- } \\
\text { Universität }\end{array}$ & staatlich & Uni \\
\hline & Kaiserslautern & $\begin{array}{l}\text { Internationales Studienkolleg der } \\
\text { Hochschule Kaiserslautern }\end{array}$ & staatlich & Uni und $\mathrm{FH}$ \\
\hline \multirow[t]{4}{*}{ Sachsen } & Dresden & TUDIAS-Studienkolleg TU Dresden & $\begin{array}{l}\text { privat (staatlich } \\
\text { anerkannt) }\end{array}$ & \\
\hline & Glauchau & Studienkolleg Glauchau & $\begin{array}{l}\text { privat (staatlich } \\
\text { anerkannt) }\end{array}$ & \\
\hline & Leipzig & Universität Leipzig Studienkolleg Sachsen & staatlich & Uni \\
\hline & Zittau & Hochschule Zittau/Görlitz Studienkolleg & staatlich & $\mathrm{FH}$ \\
\hline \multirow[t]{3}{*}{ Sachsen-Anhalt } & Halle-Merseburg & $\begin{array}{l}\text { Staatlich anerkanntes Studienkolleg Halle- } \\
\text { Merseburg an der Hochschule Merseburg (SKHM) } \\
\text { - Universitätsstudienkolleg der Ming CHENG } \\
\text { Institut GmbH }\end{array}$ & $\begin{array}{l}\text { privat (staatlich } \\
\text { anerkannt) }\end{array}$ & \\
\hline & Halle & $\begin{array}{l}\text { Studienkolleg an der Martin-Luther-Universität } \\
\text { Halle-Wittenberg (Landesstudienkolleg Sachsen- } \\
\text { Anhalt Abteilung Halle) }\end{array}$ & staatlich & Uni \\
\hline & Köthen & Studienkolleg Sachsen-Anhalt/Köthen & staatlich & Uni und $\mathrm{FH}$ \\
\hline Schleswig-Holstein & Kiel & Studienkolleg an der FH Kiel & staatlich & $\mathrm{FH}$ \\
\hline Thüringen & Nordhausen & Staatliches Studienkolleg & staatlich & Uni und $\mathrm{FH}$ \\
\hline
\end{tabular}


pauschale von $100 €$

Bewerbung direkt am Studienkolleg

$7.000 €$

2

B1

Bewerbung für einen Studiengang an der Universität

Kostenlos

Bewerbung direkt am Studienkolleg

Bewerbung direkt am Studienkolleg

Bewerbung am Studienkolleg mit Vorzulassung zum

Studium an einer Hochschule in Sachsen

\author{
1. Semester: $2.250 €$ \\ 2. Semester: $2.550 €$ \\ Aufnahmetest: $20 €$
}

Leider konnten keine Informa-

tionen zur Höhe der Studien-

gebühren gefunden werden

Bewerbung am Studienkolleg mit Vorzulassung zum

Semesterbeitrag von ca. $230 €$

Studium an einer Hochschule in Sachsen

Bewerbung für einen Studiengang an der Hochschule Zittau/Görlitz

Bewerbung am Studienkolleg mit einer bedingten Vorzulassung durch eine deutsche Universität oder FH
Ca. $6000 €$

2
Bewerbung über uni-assist für einen entsprechenden Kurs

\section{Semesterbeitrag von ca. $95 € \quad 2$}

$\mathrm{B} 1$

B1

B1
Semesterbeitrag von ca. $230 € \quad 2$

$\mathrm{B} 2$

$\begin{array}{llc}\text { Bewerbung an der Hochschule Anhalt in Köthen } & \text { Semesterbeitrag von ca.90€ } 2 \quad \text { B1 } \\ \text { Bewerbung direkt am Studienkolleg an der FH Kiel } & \begin{array}{l}\text { Semesterbeitrag von ca. 250€ } \\ \text { und 100€ Immatrikulations- } \\ \text { gebühr }\end{array} & \text { B1 }\end{array}$

Bewerbung an einer Hochschule in Thüringen. Die Semesterbeitrag von $158,58 € \quad 2$

jeweilige Hochschule entscheidet, ob der Bewerber das

Studienkolleg für die FSP oder die DSH besuchen muss. 


\title{
FRAGEBOGEN: UMFRAGE ZU STUDIENVORBEREITUNG UND -EINSTIEG INTERNATIONALER STUDIERENDER IN DEUTSCHLAND
}

\author{
Name der Hochschule* \\ Bitte geben Sie Ihre Antwort hier ein:
}

An unserer Hochschule werden studienvorbereitende Maßnahmen angeboten: *

Bitte wählen Sie nur eine der folgenden Antworten aus:
Ja
Nein

In Anlehnung an Schellhorn et al (2019) verstehen wir unter „Maßnahmen zur Studienvorbereitung“ Angebote, welche von den Hochschulen selbst oder von Partnerorganisationen initiiert werden mit dem gemeinsamen Ziel, den Studieneinstieg zu erleichtern. Sie begleiten die Studierenden bzw. Studieninteressierten vor Beginn ihres Studiums und zielen darauf $a b$, wichtige Studienbereiche und teilweise fachliche Inhalte aufzufrischen. Darüber hinaus gibt es eine Reihe von Maßnahmen, deren erfolgreiches Absolvieren sich auf den Hochschulzugang auswirkt.

Unsere Hochschule bietet folgende studienvorbereitende Maßnahmen an, welche die Studieninteressierten nutzen können, die aber keine Relevanz für ihren Zugang zum Studium haben: *

Beantworten Sie diese Frage nur, wenn folgende Bedingungen erfüllt sind:

Antwort war ,Ja' bei Frage ,2 [a2]“' (An unserer Hochschule werden studienvorbereitende Maßnahmen angeboten:)

Bitte wählen Sie alle zutreffenden Antworten aus:

Studienorientierung (Online-Self-Assessments, Studienberatung, Studienwahl)

Studienkollegs

Förderung und Überprüfung von Fachkenntnissen (z.B. Mathematik, Deutsch, Physik, Programmierung)

studienvorbereitende Sprachkurse

Studienkompetenzen (Selbstständigkeit, Zeitmanagement, Vorbereitungen auf Sprechstunden- und Prüfungsgespräche)

Mentoring-bzw. Tutorenprogramme

Software und Apps im Studium

(Vor)-Praktika

Interkulturelle Sensibilisierung (deutsche (Wissenschafts-)kultur, interkulturelle Trainings)

wissenschaftliches Arbeiten

Vorbereitung für Eingangsprüfungen

Sonstiges: 
Haben Sie studienvorbereitende Angebote bzw. Testverfahren, deren erfolgreiches Absolvieren bei der Zulassung von Studienbewerberinnen und Studienbewerbern, insbesondere ohne HZB, berücksichtigt wird?

Beantworten Sie diese Frage nur, wenn folgende Bedingungen erfüllt sind:

Antwort war ,Ja' bei Frage ,2 [a2]“ (An unserer Hochschule werden studienvorbereitende Maßnahmen angeboten:)

Bitte wählen Sie alle zutreffenden Antworten aus:

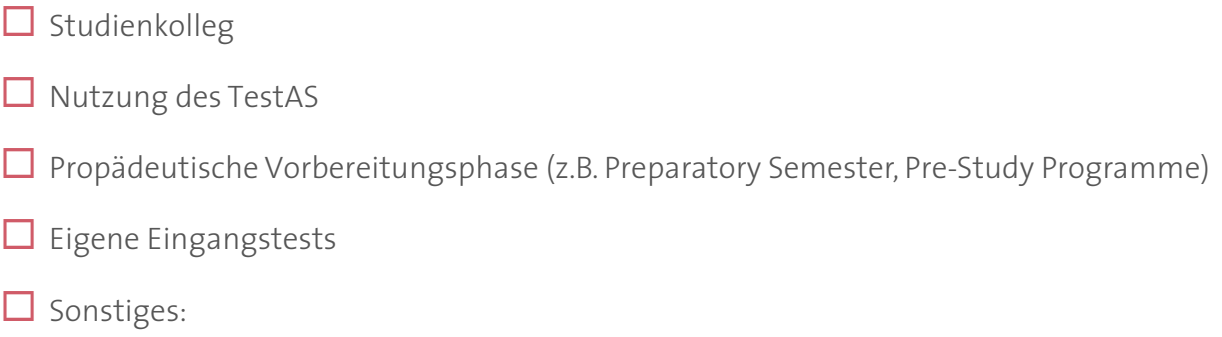

Sind diese Angebote bzw. Maßnahmen freiwillig oder obligatorisch?*

Beantworten Sie diese Frage nur, wenn folgende Bedingungen erfüllt sind:

Antwort war ,Ja' bei Frage ,2 [a2]’ (An unserer Hochschule werden studienvorbereitende Maßnahmen angeboten:)

Bitte wählen Sie die zutreffende Antwort für jeden Punkt aus:

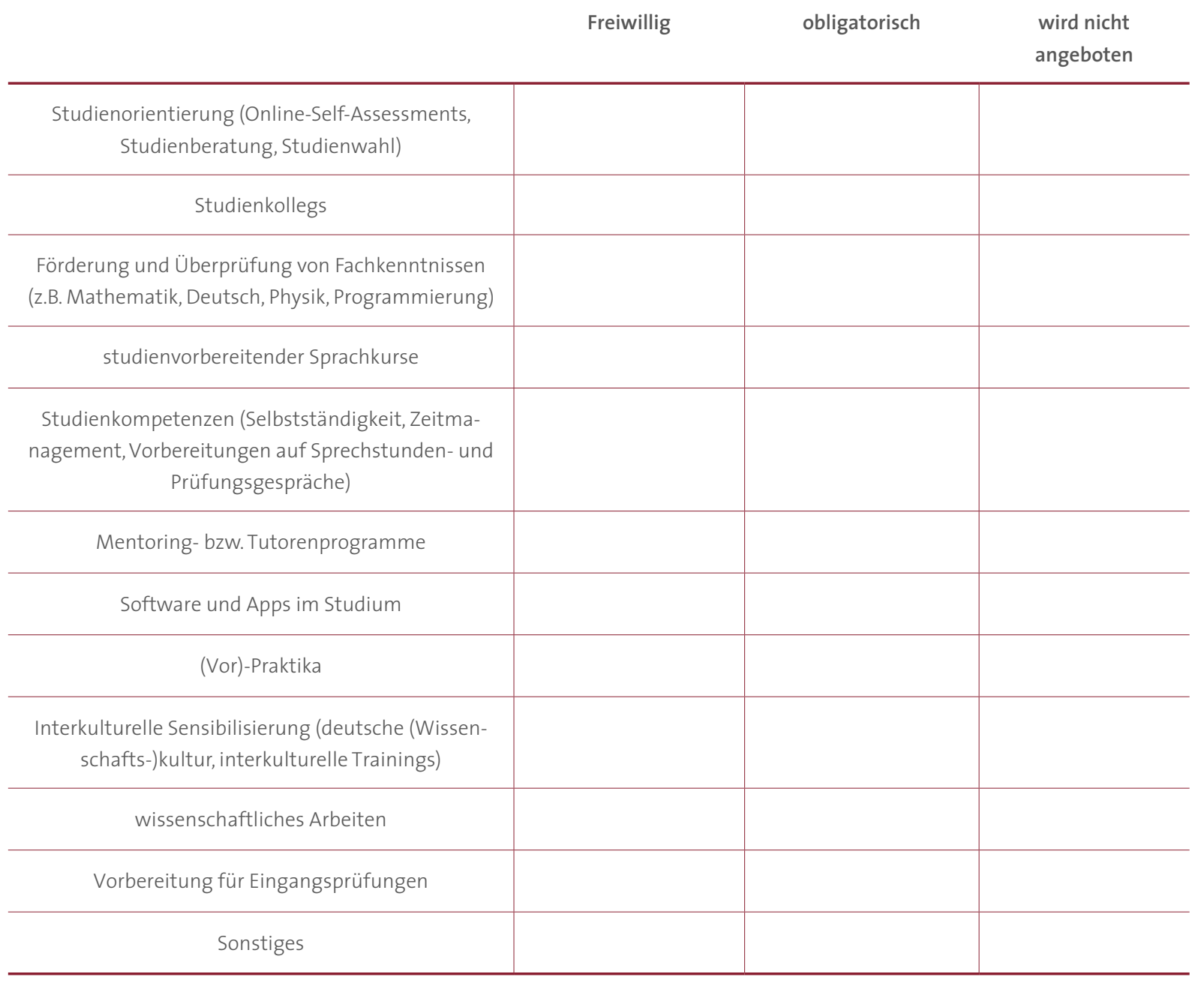


Sind diese Angebote bzw. Maßnahmen kostenlos oder kostenpflichtig? *

Beantworten Sie diese Frage nur, wenn folgende Bedingungen erfüllt sind:

Antwort war ,Ja' bei Frage ,2 [a2]' (An unserer Hochschule werden studienvorbereitende Maßnahmen angeboten:)

Bitte wählen Sie die zutreffende Antwort für jeden Punkt aus:

Kostenlos

kostenpflichtig

wird nicht

angeboten

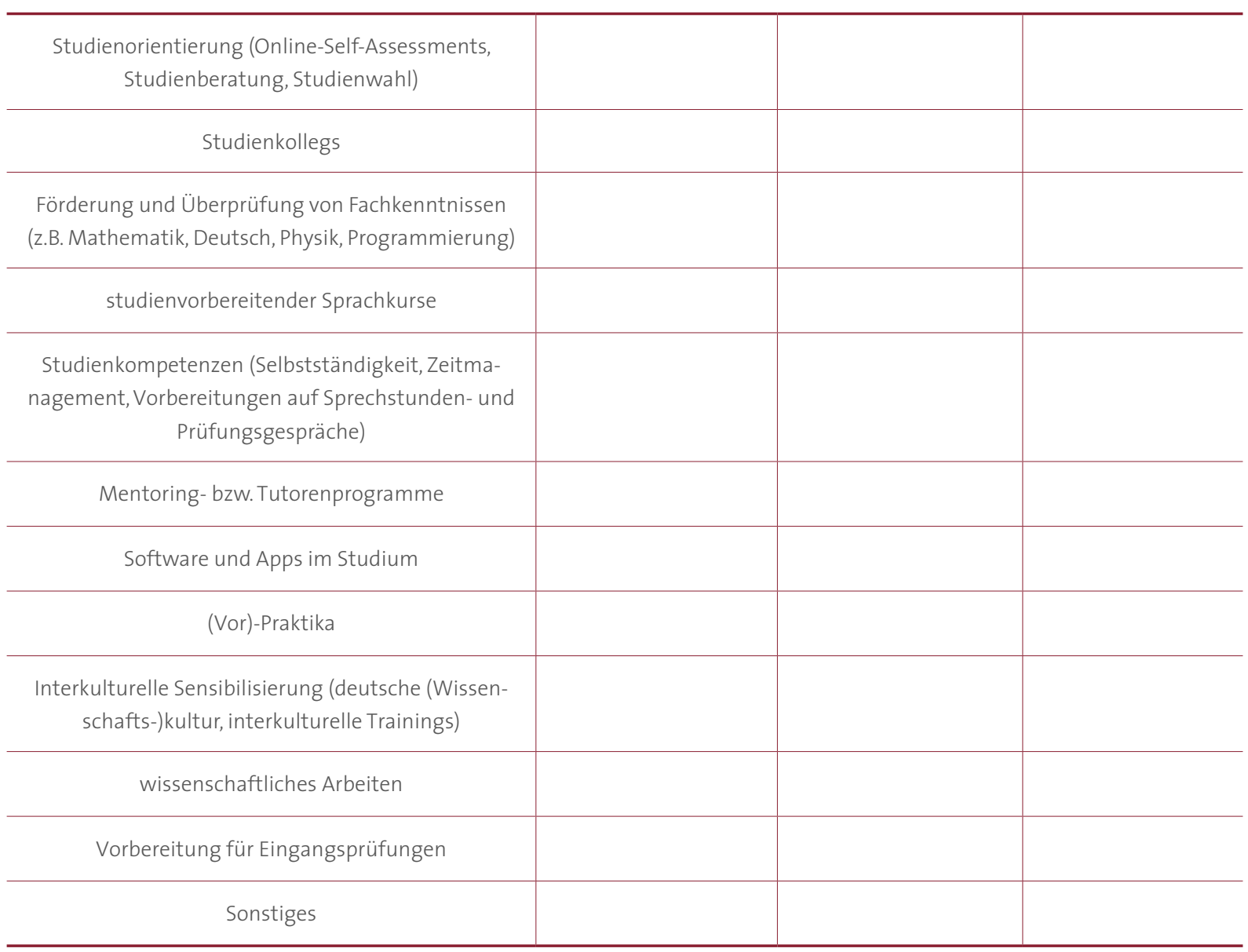

An unserer Hochschule werden folgende Formate angeboten:

Beantworten Sie diese Frage nur, wenn folgende Bedingungen erfüllt sind:

Antwort war ,Ja‘ bei Frage ,2 [a2]“ (An unserer Hochschule werden studienvorbereitende Maßnahmen angeboten: )

Bitte wählen Sie alle zutreffenden Antworten aus:

Digital

Integriertes Lernen (Blended Learning)

Präsenz 
Gibt es für die studienvorbereitende/n Maßnahme/n Websiten mit weiteren Informationen? *

Beantworten Sie diese Frage nur, wenn folgende Bedingungen erfüllt sind:

Antwort war ,Ja' bei Frage ,2 [a2]' (An unserer Hochschule werden studienvorbereitende Maßnahmen angeboten:)

Bitte wählen Sie nur eine der folgenden Antworten aus:

$\square$ Ja

$\square$ Nein

Fügen Sie uns bitte die Links zu der Website bzw. den Websiten mit weiterführenden Informationen ein:*

Beantworten Sie diese Frage nur, wenn folgende Bedingungen erfüllt sind:

Antwort war ,Ja' bei Frage ,2 [a2]“ (An unserer Hochschule werden studienvorbereitende Maßnahmen angeboten:) und Antwort war ,Ja' bei Frage ,8 [a5]' (Gibt es für die studienvorbereitende/n Maßnahme/n Websiten mit weiteren Informationen?)

Bitte geben Sie Ihre Antwort hier ein:

Fügen Sie uns bitte eine kurze Beschreibung bzw. Beschreibungen der studienvorbereitenden Maßnahmen ein (Beschreibung Maßnahme, ggf. Kosten Kurs, Teilnehmergröße)

Beantworten Sie diese Frage nur, wenn folgende Bedingungen erfüllt sind:

Antwort war ,Ja' bei Frage ,2 [a2] ' (An unserer Hochschule werden studienvorbereitende Maßnahmen angeboten:) und Antwort war ,Nein' bei Frage, 8 [a5]' (Gibt es für die studienvorbereitende/n Maßnahme/n Websiten mit weiteren Informationen?)

Bitte geben Sie Ihre Antwort hier ein: 
Wer ist an Ihrer Hochschule für die studienvorbereitenden Maßnahmen zuständig? *

Beantworten Sie diese Frage nur, wenn folgende Bedingungen erfüllt sind:

Antwort war ,Ja' bei Frage ,2 [a2]' (An unserer Hochschule werden studienvorbereitende Maßnahmen angeboten:)

Bitte wählen Sie alle zutreffenden Antworten aus:

Studienkolleg

International Office/ Akademisches Auslandsamt

Studierendenwerk

Studierendenberatung

Sprachzentrum

Studierendensekretariat

Fakultät bzw. Fachbereich (gerne bei sonstiges ergänzen welche)

Sonstiges:

Konzentrieren sich Ihre studienvorbereitenden Maßnahmen auf eine bestimmte Zielgruppe? *

Beantworten Sie diese Frage nur, wenn folgende Bedingungen erfüllt sind:

Antwort war , Ja' bei Frage ,2 [a2]' (An unserer Hochschule werden studienvorbereitende Maßnahmen angeboten:)

Bitte wählen Sie nur eine der folgenden Antworten aus:

$\mathrm{Ja}$

Nein

Auf welche Zielgruppe(n) konzentrieren sich Ihre studienvorbereitenden Maßnahmen? *

Beantworten Sie diese Frage nur, wenn folgende Bedingungen erfüllt sind:

Antwort war ,Ja' bei Frage, 2 [a2] (An unserer Hochschule werden studienvorbereitende Maßnahmen angeboten:) und Antwort war ,Ja' bei Frage, 12 [a9]' (Konzentrieren sich Ihre studienvorbereitenden Maßnahmen auf eine bestimmte Zielgruppe?)

Kommentieren wenn eine Antwort gewählt wird 
Bitte wählen Sie die zutreffenden Punkte aus und schreiben Sie einen Kommentar dazu:

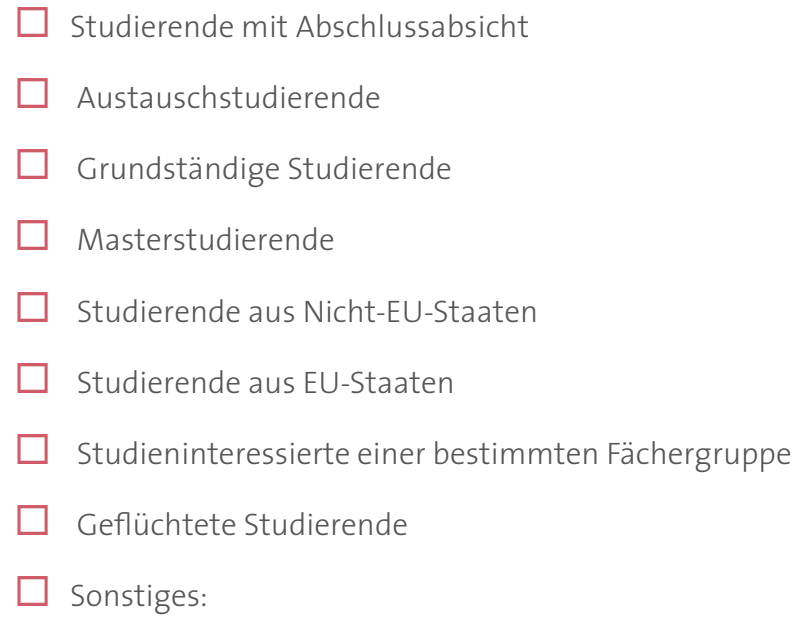

Haben Sie vor studienvorbereitende Maßnahmen an Ihrer Hochschule einzuführen? Falls ja, welche? * Beantworten Sie diese Frage nur, wenn folgende Bedingungen erfüllt sind: Antwort war ,Nein' bei Frage ,2 [a2]' (An unserer Hochschule werden studienvorbereitende Maßnahmen angeboten:) Bitte geben Sie Ihre Antwort hier ein:

Existieren Herausforderungen oder Hindernisse bzw. Bedarfe im Bereich der studienvorbereitenden Maßnahmen, die Sie hier vonseiten der Hochschule formulieren möchten?

Bitte geben Sie Ihre Antwort hier ein: 
Name der Kontaktperson für Rückfragen:*

Bitte geben Sie Ihre Antwort(en) hier ein:

- Vorname

- Nachname

Kontaktdaten für Rückfragen: *

Bitte geben Sie Ihre Antwort(en) hier ein:

- Telefonnummer

- Mailadresse

Wir möchten uns herzlich bei Ihnen für Ihre Teilnahme bedanken! Sie haben nun die Möglichkeit, uns noch etwas mitzuteilen, falls aus Ihrer Sicht in der Umfrage etwas Wichtiges nicht zur Sprache kam.

Bitte geben Sie Ihre Antwort hier ein: 


\section{$\triangle \Delta \triangle D \quad \begin{aligned} & \text { Deutscher Akademischer Austauschdienst } \\ & \text { German Academic Exchange Service }\end{aligned}$}

Der Deutsche Akademische Austauschdienst (DAAD) ist die Organisation der deutschen Hochschulen und ihrer Studierenden zur Internationalisierung des Wissenschaftssystems. Er schafft Zugänge zu den besten Studien- und Forschungsmöglichkeiten für Studierende, Forschende und Lehrende durch die Vergabe von Stipendien. Der DAAD fördert transnationale Kooperationen und Partnerschaften zwischen Hochschulen und ist die Nationale Agentur für die europäische Hochschulzusammenarbeit; zu diesem Zweck unterhält er ein weltweites Netzwerk mit über 70 Auslandsbüros und rund 450 Lektorate weltweit sowie die internationale DAAD-Akademie (iDA). 2017 hat der DAAD rund 140.000 Deutsche und Ausländer rund um den Globus gefördert. Der DAAD wird überwiegend aus Mitteln des Auswärtigen Amts, des Bundesministeriums für Bildung und Forschung, des Bundesministeriums für wirtschaftliche Zusammenarbeit und Entwicklung und der Europäischen Union finanziert. 